\title{
Mean Field Games: Numerical Methods
}

\author{
Yves Achdou, Italo Capuzzo-Dolcetta ${ }^{\dagger}$
}

May 2, 2009

\begin{abstract}
Mean field type models describing the limiting behavior of stochastic differential game problems, as the number of players tends to $+\infty$, have recently been introduced by J-M. Lasry and P-L. Lions. Numerical methods for the approximation of stationary and nonstationary such models are proposed. In particular, existence and uniqueness are investigated, as well as bounds on the solutions. Numerical experiments are carried out.
\end{abstract}

\section{Introduction}

Mean field type models describing the limiting behavior of stochastic differential game problems as the number of players tends to $+\infty$ have recently been introduced by J-M. Lasry and P-L. Lions $[11,12,13]$. In the stationary setting, a typical model of this kind comprises the following system:

$$
\begin{aligned}
-\nu \Delta u+H(x, \nabla u)+\lambda & =V[m], x \in \mathbb{T}^{d}, \\
-\nu \Delta m-\operatorname{div}\left(m \frac{\partial H}{\partial p}(x, \nabla u)\right) & =0, x \in \mathbb{T}^{d}, \\
\int_{\mathbb{T}^{d}} u=0, \quad \int_{\mathbb{T}^{d}} m & =1, \quad m>0 .
\end{aligned}
$$

The unknowns are the scalar functions $u, m$ defined on the $d$-dimensional torus $\mathbb{T}^{d}$ and the real number $\lambda$. The data are a positive number $\nu$, the Hamiltonian $H: \mathbb{T}^{d} \times \mathbb{R}^{d} \rightarrow \mathbb{R}$, convex with respect to $p$ and the (nonlinear) mapping $V$ associating to a probability density $m$ a Lipschitz function $V[m]$ on $\mathbb{T}^{d}$. Typical examples for $V$ include nonlocal smoothing operators.

The time-dependent analogue of system (1)-(3), also considered in $[11,12,13]$, is

$$
\begin{aligned}
\frac{\partial u}{\partial t}-\nu \Delta u+H(x, \nabla u) & =V[m], \quad \text { in } \mathbb{T}^{d} \times(0, T), \\
\frac{\partial m}{\partial t}+\nu \Delta m+\operatorname{div}\left(m \frac{\partial H}{\partial p}(x, \nabla u)\right) & =0, \quad \text { in } \mathbb{T}^{d} \times(0, T), \\
\int_{\mathbb{T}^{d}} m(x, t) d x=1, \quad m>0, & \\
u(t=0)=V_{0}[m(t=0)], \quad m(t=T) & =m_{0} .
\end{aligned}
$$

\footnotetext{
*UFR Mathématiques, Université Paris 7, Case 7012, 175 rue du Chevaleret, 75013 Paris, France and UMR 7598, Laboratoire Jacques-Louis Lions, F-75005, Paris, France. achdou@math.jussieu.fr

${ }^{\dagger}$ Dipartimento di Matematica, Università Roma "La Sapienza", Piazzale A. Moro 2, I-00185 Roma, capuzzo@mat.uniroma1.it
} 
We refer to the mentioned papers of J-M. Lasry and P-L. Lions for analytical results concerning system (1)-(3) as well as for its interpretation in stochastic game theory. Let us only mention here that a very important feature of the mean field model above is that uniqueness and stability may be obtained under reasonable assumptions, see [11, 12, 13], in contrast with the Nash system describing the individual behavior of each player, for which uniqueness hardly occurs.

The aim of the present work is to propose discrete approximations by finite difference methods of the mean field model, both in the stationary case (1)-(3) or the non-stationary one (4)-(7). The numerical schemes that we use rely basically on monotone approximations of the Hamiltonian and on a suitable weak formulation of the equation for $m$. For the sake of simplicity of notations, we will always consider the 2-dimensional case although our approach and results hold for general $d$.

These schemes have several important features:

- existence and uniqueness for the discretized problems can be obtained by similar arguments as those used in the continuous case,

- they are robust when $\nu \rightarrow 0$ (the deterministic limit of the models),

- bounds on the solutions, which are uniform in the grid step, can be proved under reasonable assumptions on the data.

Let us mention in this respect that an important research activity is currently going on about approximation procedures for mean field games. Quite recently, we learned about a different numerical approach, based on the reformulation of the model as an optimization problem, which is restricted however to the case when $V[m](x)=g(m(x))$, see [10]. See also [5] for a very recent work on discrete time, finite state space mean field games.

In Section 2, we present the approximation of the nonlinear operators involved in e.g. (1)-(3) and the main assumptions that are going to be made. The finite difference scheme for the stationary model is discussed in Section 3: emphasis is put on existence, uniqueness and on bounds on the solution; the main difficulty faced there is to obtain bounds on the solution which are uniform in the discretization parameters. An example of a convergence result is also supplied in $\S 3$.

In Section 4 we discuss an implicit in time finite difference method for the following evolution problem comprising two forward parabolic equations:

$$
\begin{aligned}
\frac{\partial u}{\partial t}-\nu \Delta u+H(x, \nabla u) & =V[m], \quad \text { in } \mathbb{T}^{2} \times(0, T), \\
\frac{\partial m}{\partial t}-\nu \Delta m-\operatorname{div}\left(m \frac{\partial H}{\partial p}(x, \nabla u)\right) & =0, \quad \text { in } \mathbb{T}^{2} \times(0, T), \\
\int_{\mathbb{T}^{2}} m(x, t) d x=1, \quad m>0, & \\
u(x, 0)=u_{0}(x), \quad m(x, 0) & =m_{0}(x) .
\end{aligned}
$$

By analogy with known results long time approximations for the cell problem in homogenization see [14], we expect that there exists some $\bar{\lambda} \in \mathbb{R}$ such that $u(x, t)-\bar{\lambda} t$ and $m(x, t)$ converge, as $t \rightarrow+\infty$, to the solution $(\bar{u}(x), \bar{m}(x), \bar{\lambda})$ of the stationary system (1)-(3). The main result in Section 4 is Theorem 5 on the existence for the discrete system.

Section 5 deals with the non-stationary mean field system (4)-(7) and contains results on existence and uniqueness.

Finally, the long time strategy mentioned above and numerical experiments for the stationary models are described in $\S 6$.

A Newton method for the non-stationary problem (4)-(7) will be discussed in a forthcoming work, [2]. 


\section{The finite difference operators}

\subsection{The proposed schemes}

For simplicity, we discuss the approximation of (1)-(3).

Let $\mathbb{T}_{h}^{2}$ be a uniform grid on the torus with mesh step $h$, (assuming that $1 / h$ is an integer $N_{h}$ ), and $x_{i j}$ denote a generic point in $\mathbb{T}_{h}^{2}$. The values of $u$ and $m$ at $x_{i, j}$ will respectively be approximated by $U_{i, j}$ and $M_{i, j}$.

Hereafter, we will often make the following assumptions on the operator $V$ :

$\left(\mathbf{A}_{1}\right): V: m \rightarrow V[m]$ maps the set of probability measures into a bounded set of Lipschitz functions on $\mathbb{T}^{2}$.

$\left(\mathbf{A}_{\mathbf{2}}\right)$ : if $m_{n}$ converges weakly to $m$ then $V\left[m_{n}\right]$ converges to $V[m]$ uniformly on $\mathbb{T}^{2}$.

A possible approximation of $V[m]\left(x_{i, j}\right)$ is

$$
\left(V_{h}[M]\right)_{i, j}=V\left[m_{h}\right]\left(x_{i, j}\right)
$$

calling $m_{h}$ the piecewise constant function taking the value $M_{i, j}$ in the square $\left|x-x_{i, j}\right|_{\infty} \leq h / 2$, and assuming that $V\left[m_{h}\right]$ can be computed in practice.

We introduce the finite difference operators

$$
\left(D_{1}^{+} U\right)_{i, j}=\frac{U_{i+1, j}-U_{i, j}}{h} \text { and } \quad\left(D_{2}^{+} U\right)_{i, j}=\frac{U_{i, j+1}-U_{i, j}}{h}
$$

and the numerical Hamiltonian $g: \mathbb{T}^{2} \times \mathbb{R}^{4} \rightarrow \mathbb{R}$. The finite difference approximation of $H(x, \nabla u)$ is $g\left(x_{i, j},\left(D_{1}^{+} U^{n+1}\right)_{i, j},\left(D_{1}^{+} U^{n+1}\right)_{i-1, j},\left(D_{2}^{+} U^{n+1}\right)_{i, j},\left(D_{2}^{+} U^{n+1}\right)_{i, j-1}\right)$. Classically, we choose the discrete version of $(1)$ as

$$
\left(\begin{array}{l}
\frac{\nu}{h^{2}}\left(4 U_{i, j}-U_{i+1, j}-U_{i-1, j}-U_{i, j+1}-U_{i, j-1}\right) \\
+g\left(x_{i, j},\left(D_{1}^{+} U\right)_{i, j},\left(D_{1}^{+} U\right)_{i-1, j},\left(D_{2}^{+} U\right)_{i, j},\left(D_{2}^{+} U\right)_{i, j-1}\right)+\lambda_{h}
\end{array}\right)=\left(V_{h}[M]\right)_{i, j} .
$$

With the notations

$$
\left(\Delta_{h} W\right)_{i, j}=-\frac{1}{h^{2}}\left(4 W_{i, j}-W_{i+1, j}-W_{i-1, j}-W_{i, j+1}-W_{i, j-1}\right)
$$

and

(14) becomes

$$
\left[D_{h} W\right]_{i, j}=\left(\left(D_{1}^{+} W\right)_{i, j},\left(D_{1}^{+} W\right)_{i-1, j},\left(D_{2}^{+} W\right)_{i, j},\left(D_{2}^{+} W\right)_{i, j-1}\right)^{T},
$$

$$
-\nu\left(\Delta_{h} U\right)_{i, j}+g\left(x_{i, j},\left[D_{h} U\right]_{i, j}\right)=\left(V_{h}[M]\right)_{i, j} .
$$

The following assumptions on the discrete Hamiltonian $g:\left(q_{1}, q_{2}, q_{3}, q_{4}\right) \rightarrow g\left(x, q_{1}, q_{2}, q_{3}, q_{4}\right)$ will be made

$\left(\mathbf{H}_{1}\right)$ : monotonicity: $g$ is nonincreasing with respect to $q_{1}$ and $q_{3}$ and nondecreasing with respect to $q_{2}$ and $q_{4}$.

$\left(\mathbf{H}_{2}\right)$ : consistency:

$$
g\left(x, q_{1}, q_{1}, q_{2}, q_{2}\right)=H(x, q), \quad \forall x \in \mathbb{T}^{2}, \forall q=\left(q_{1}, q_{2}\right) \in \mathbb{R}^{2} .
$$

$\left(\mathbf{H}_{3}\right)$ Differentiability: $g$ is of class $\mathcal{C}^{1}$.

The discrete version of (2) is chosen according to the following heuristics: 
- when $u$ is fixed, (2) is a linear elliptic equation for $m$ : therefore, when $U$ is fixed, the discrete version of (2) should yield a matrix with the following properties:

- the diagonal coefficients are positive,

- the off-diagonal coefficients are nonpositive,

so that hopefully a discrete maximum principle may be used.

- The argument used by Lasry and Lions for the uniqueness of the continuous problems (1)-(3) and (4)-(7) (see [13, 11, 12]) should hold in the discrete cases. For this reason, the discrete Hamiltonian $g$ introduced above should be used in the discrete version of (2) as well, and we will make another assumption on $g$ :

$\left(\mathbf{H}_{4}\right)$ : convexity : the function $\left(q_{1}, q_{2}, q_{3}, q_{4}\right) \rightarrow g\left(x, q_{1}, q_{2}, q_{3}, q_{4}\right)$ is convex.

The main idea is to consider the weak form of (2): it involves the term

$$
-\int_{\mathbb{T}^{2}} \operatorname{div}\left(m \frac{\partial H}{\partial p}(x, \nabla u)\right) w=\int_{\mathbb{T}^{2}} m \frac{\partial H}{\partial p}(x, \nabla u) \cdot \nabla w
$$

which will be approximated by

$$
h^{2} \sum_{i, j} m_{i, j} \nabla_{q} g\left(x_{i, j},\left[D_{h} U\right]_{i, j}\right) \cdot\left[D_{h} W\right]_{i, j} .
$$

This yields the following discrete version of (9):

$$
\left.\begin{array}{rl}
-\nu\left(\Delta_{h} M\right)_{i, j}- & -\frac{1}{h}\left(\begin{array}{c}
M_{i, j} \frac{\partial g}{\partial q_{1}}\left(x_{i, j},\left[D_{h} U\right]_{i, j}\right)-M_{i-1, j} \frac{\partial g}{\partial q_{1}}\left(x_{i-1, j},\left[D_{h} U\right]_{i-1, j}\right) \\
+M_{i+1, j} \frac{\partial g}{\partial q_{2}}\left(x_{i+1, j},\left[D_{h} U\right]_{i+1, j}\right)-M_{i, j} \frac{\partial g}{\partial q_{2}}\left(x_{i, j},\left[D_{h} U\right]_{i, j}\right)
\end{array}\right) \\
- & -\frac{1}{h}\left(\begin{array}{c}
M_{i, j} \frac{\partial g}{\partial q_{3}}\left(x_{i, j},\left[D_{h} U\right]_{i, j}\right)-M_{i, j-1} \frac{\partial g}{\partial q_{3}}\left(x_{i, j-1},\left[D_{h} U\right]_{i, j-1}\right) \\
+M_{i, j+1} \frac{\partial g}{\partial q_{4}}\left(x_{i, j+1},\left[D_{h} U\right]_{i, j+1}\right)-M_{i, j} \frac{\partial g}{\partial q_{4}}\left(x_{i, j},\left[D_{h} U\right]_{i, j}\right)
\end{array}\right)
\end{array}\right\}=0 .
$$

We will also use the more compact but less explicit notation:

$$
\mathcal{B}_{i, j}(U, M)=\frac{1}{h}\left(\begin{array}{c}
\left(\begin{array}{c}
M_{i, j} \frac{\partial g}{\partial q_{1}}\left(x_{i, j},\left[D_{h} U\right]_{i, j}\right)-M_{i-1, j} \frac{\partial g}{\partial q_{1}}\left(x_{i-1, j},\left[D_{h} U\right]_{i-1, j}\right) \\
+M_{i+1, j} \frac{\partial g}{\partial q_{2}}\left(x_{i+1, j},\left[D_{h} U\right]_{i+1, j}\right)-M_{i, j} \frac{\partial g}{\partial q_{2}}\left(x_{i, j},\left[D_{h} U\right]_{i, j}\right)
\end{array}\right) \\
+\left(\begin{array}{c}
M_{i, j} \frac{\partial g}{\partial q_{3}}\left(x_{i, j},\left[D_{h} U\right]_{i, j}\right)-M_{i, j-1} \frac{\partial g}{\partial q_{3}}\left(x_{i, j-1},\left[D_{h} U\right]_{i, j-1}\right) \\
+M_{i, j+1} \frac{\partial g}{\partial q_{4}}\left(x_{i, j+1},\left[D_{h} U\right]_{i, j+1}\right)-M_{i, j} \frac{\partial g}{\partial q_{4}}\left(x_{i, j},\left[D_{h} U\right]_{i, j}\right)
\end{array}\right)
\end{array}\right) .
$$

This yields the shorter form of (19):

$$
-\nu\left(\Delta_{h} M\right)_{i, j}-\mathcal{B}_{i, j}(U, M)=0 .
$$

Remark 1 It is important to realize that the operator $M \mapsto-\nu\left(\Delta_{h} M\right)_{i, j}-\mathcal{B}_{i, j}(U, M)$ is the adjoint of the linearized version of the operator $U \mapsto-\nu\left(\Delta_{h} U\right)_{i, j}+g\left(x_{i, j},\left[D_{h} U\right]_{i, j}\right)$. 
$\left(\mathbf{H}_{5}\right)$ a further consistency assumption One has to assume that the discrete operator in (21) is a consistent approximation of the differential operator in (2), i.e. that there exists a positive integer $\ell$, a real number $\delta_{0}, \delta_{0} \in(0,1)$ and some positive real number $r$ such that for every $v, m \in \mathcal{C}^{\ell, \delta_{0}}\left(\mathbb{T}^{2}\right)$, there is a constant $\bar{K}$ depending on the norms of $v$ and $m$ in the previously mentioned Schauder spaces, such that for all $h<1$, calling $\widetilde{V}$ and $\widetilde{M}$ the grid functions defined by $\widetilde{V}_{i, j}=\frac{1}{h^{2}} \int_{\left|x-x_{i, j}\right|_{\infty}<h / 2} v d x$ and $\widetilde{M}_{i, j}=\frac{1}{h^{2}} \int_{\left|x-x_{i, j}\right|_{\infty}<h / 2} m d x$, we have for all $i, j$,

$$
\left|\mathcal{B}_{i, j}(\widetilde{V}, \widetilde{M})-\operatorname{div}\left(m \frac{\partial H}{\partial p}(x, \nabla v)\right)\left(x_{i, j}\right)\right| \leq \bar{K} h^{r}
$$

This assumption is clearly fulfilled if $g$ satisfies (18) and if $g$ and $H$ are smooth enough.

\subsection{Summary}

Finally, the finite difference approximation of (1)-(3) is to look for two grid functions $U, M$ on $\mathbb{T}_{h}^{2}$ and for a scalar $\lambda$ such that

$$
\begin{aligned}
& \forall i, j: 0 \leq i, j<N_{h}, \quad\left\{\begin{aligned}
-\nu\left(\Delta_{h} U\right)_{i, j}+g\left(x_{i, j},\left[D_{h} U\right]_{i, j}\right)+\lambda & =\left(V_{h}[M]\right)_{i, j}, \\
-\nu\left(\Delta_{h} M\right)_{i, j}-\mathcal{B}_{i, j}(U, M) & =0,
\end{aligned}\right. \\
& \sum_{i, j} U_{i, j}=0 \\
& h^{2} \sum_{i, j} M_{i, j}=1 \quad \text { and } \quad M_{i, j} \geq 0 \text { for } 0 \leq i, j<N_{h} .
\end{aligned}
$$

with $\left(V_{h}[M]\right)_{i, j}$ defined in $(12), \mathcal{B}_{i, j}(U, M)$ defined in (20) and the numerical Hamiltonian $g$ : $\mathbb{T}^{2} \times \mathbb{R}^{4} \rightarrow \mathbb{R}$ satisfying at least $\left(\mathbf{H}_{\mathbf{1}}\right)$-( $\left.\mathbf{H}_{\mathbf{3}}\right)$ above.

The same strategy will be used to approximate the nonstationary problems (4)-(7) and (8)-(11) with implicit schemes, see respectively (86)-(88) and (65)-(66) below.

In what follows, for two grid functions $W$ and $Z$ on $\mathbb{T}_{h}^{2}$, denote by $(W, Z)_{2}$ the inner product $(W, Z)_{2}=\sum_{i, j} W_{i, j} Z_{i, j}$.

\subsection{A useful lemma}

We recall a useful lemma, which can be found in e.g. [4]. We give its proof for completeness.

Lemma 1 Let $V$ be a grid function on $\mathbb{T}_{h}^{2}$ and $\rho$ be a positive parameter. Assume that $g$ satisfies $\left(\mathbf{H}_{\mathbf{1}}\right)-\left(\mathbf{H}_{\mathbf{3}}\right)$. There exists a unique grid function $U$ such that

$$
\rho U_{i, j}+g\left(x_{i, j},\left[D_{h} U\right]_{i, j}\right)-\nu\left(\Delta_{h} U\right)_{i, j}=V_{i, j} .
$$

Proof. Existence for (26) is proved by using Leray-Schauder fixed point theorem: indeed, we consider the mapping $\mathcal{F}: \mathbb{R}^{N_{h}^{2}} \mapsto \mathbb{R}_{h}^{N_{h}^{2}}$,

$$
(\mathcal{F}(U))_{i, j}=\frac{1}{\rho}\left(\nu\left(\Delta_{h} U\right)_{i, j}-g\left(x_{i, j},\left[D_{h} U\right]_{i, j}\right)+V_{i, j}\right),
$$

and the real number $r=\max _{(i, j)} \frac{1}{\rho}\left|H\left(x_{i, j}, 0\right)-V_{i, j}\right|$. From the continuity of $g, \mathcal{F}$ is continuous from $B_{r}=\left\{U \in \mathbb{R}^{N_{h}^{2}}:\|U\|_{\infty} \leq r\right\}$ to $\mathbb{R}^{N_{h}^{2}}$.

Assuming that $U \in \partial B_{r}$, there must exist at least one pair of indices $\left(i_{0}, j_{0}\right)$ such that $U_{i_{0}, j_{0}}= \pm r$. Assuming that $U_{i_{0}, j_{0}}=r$, we have

$$
\nu\left(\Delta_{h} U\right)_{i_{0}, j_{0}}-g\left(x_{i_{0}, j_{0}},\left[D_{h} U\right]_{i_{0}, j_{0}}\right) \leq-H\left(x_{i_{0}, j_{0}}, 0\right),
$$


from the monotonicity and the consistency of $g$. Hence,

$$
(\mathcal{F}(U))_{i_{0}, j_{0}} \leq \frac{1}{\rho}\left(-H\left(x_{i_{0}, j_{0}}, 0\right)+V_{i_{0}, j_{0}}\right) \leq r
$$

and $(\mathcal{F}(U))_{i_{0}, j_{0}} \neq \lambda U_{i_{0}, j_{0}}$ whenever $\lambda>1$. Similarly, if $U_{i_{0}, j_{0}}=-r$, then $(\mathcal{F}(U))_{i_{0}, j_{0}} \geq-r$ which implies that $(\mathcal{F}(U))_{i_{0}, j_{0}} \neq \lambda U_{i_{0}, j_{0}}$. Therefore $\mathcal{F}(U) \neq \lambda U$ for all $\lambda>1$ and $U \in \partial B_{r}$. The Leray-Schauder fixed point theorem can be used: there exists a solution of (26) in $B_{r}$. Uniqueness for (26) stems from the monotonicity of $g$.

\section{Numerical analysis of the stationary problem (23)-(25)}

Existence results for (23)-(25) can be proved under additional assumptions on $g$ and $V_{h}$. The proof strategy will be to apply Brouwer theorem to a map $\chi$ defined on the compact and convex set

$$
\mathcal{K}=\left\{\left(M_{i, j}\right)_{0 \leq i, j<N_{h}}: h^{2} \sum_{i, j} M_{i, j}=1, M_{i, j} \geq 0\right\}
$$

which can be viewed as the set of the discrete probability measures.

We will see below that existence can be proved without bounds on $U$ uniform with respect to $h$ since the problem is finite dimensional. However, when possible, we will insist much on obtaining such bounds, for example, equicontinuity with respect to $h$, because they are important for passing to the limit when $h \rightarrow 0$.

We first define a map $\Phi: M \in \mathcal{K} \rightarrow U$ where $(U, \lambda)$ is the unique solution of the first equation in (23) subject to the constraint in (24). The map $M \rightarrow \chi(M)$ is then obtained by solving a perturbation of the second equation in (23) with $U=\Phi(M)$, subject to the constraints in (25). The discrete function $U=\Phi(M)$ will be obtained by passing to the limit in the following Hamilton-Jacobi-Bellman equation

$$
\rho U_{i, j}^{(\rho)}+g\left(x_{i, j},\left[D_{h} U^{(\rho)}\right]_{i, j}\right)-\nu\left(\Delta_{h} U^{(\rho)}\right)_{i, j}=\left(V_{h}[M]\right)_{i, j},
$$

when the positive parameter $\rho$ tends to 0 . Such a strategy is reminiscent of those used for solving the cell problems in the homogenization of Hamilton-Jacobi-Bellman equations, see e.g. $[14,1,3]$. We first need to study $(28)$ and obtain some bounds on $U^{\rho}$ uniform w.r.t. $\rho$ and $M$ (and possibly uniform w.r.t. $h$ ); these will yield bounds on $U$ uniform w.r.t. $M$ (and possibly uniform w.r.t. $h)$.

\subsection{Preliminary results}

Concerning the continuous problem, one of the assumptions made in $[13,11]$ was that there exists $\theta \in(0,1)$ such that for $|p|$ large,

$$
\inf _{x \in \mathbb{T}^{2}}\left(\nabla_{x} H \cdot p+\frac{\theta}{2 \nu} H^{2}\right)>0 .
$$

It was useful in order to apply Bernstein's method to (1) and get a bound on $\|\nabla u\|_{\infty}$.

With assumption (29), we were not able to extend the method of Bernstein to the discrete level. Several other assumptions on $H$ and $g$ can be made. Assumption 1 below will make it possible to use the results of Kuo and Trudinger [9] and [8] on Hölder estimates for the solution of (28). 
The slightly stronger assumption 2 will make it possible to apply recent results of Krylov [7]. We will use the following notation:

$$
\begin{aligned}
& \left(D_{1}^{c} U\right)_{i, j}=\frac{U_{i+1, j}-U_{i-1, j}}{2 h}=\frac{1}{2}\left(\left(D_{1}^{+} U\right)_{i, j}+\left(D_{1}^{+} U\right)_{i-1, j}\right), \\
& \left(D_{2}^{c} U\right)_{i, j}=\frac{U_{i, j+1}-U_{i, j-1}}{2 h}=\frac{1}{2}\left(\left(D_{2}^{+} U\right)_{i, j}+\left(D_{2}^{+} U\right)_{i, j-1}\right), \\
& \left(D_{1}^{2} U\right)_{i, j}=\frac{U_{i+1, j}+U_{i-1, j}-2 U_{i, j}}{h^{2}}=\frac{1}{h}\left(\left(D_{1}^{+} U\right)_{i, j}-\left(D_{1}^{+} U\right)_{i-1, j}\right), \\
& \left(D_{2}^{2} U\right)_{i, j}=\frac{U_{i, j+1}+U_{i, j-1}-2 U_{i, j}}{h^{2}}=\frac{1}{h}\left(\left(D_{2}^{+} U\right)_{i, j}-\left(D_{2}^{+} U\right)_{i, j-1}\right) .
\end{aligned}
$$

Assumption 1 a) The Hamiltonian $H$ is of the form

$$
H(x, p)=\max _{\alpha \in \mathcal{A}}(p \cdot \alpha-L(x, \alpha)),
$$

where $\mathcal{A}$ is a compact subset of $\mathbb{R}^{2}$ and $L$ is a $\mathcal{C}^{0}$ function on $\mathbb{T}^{2} \times \mathcal{A}$. The function $H$ is continuous with respect to $x$ and of class $\mathcal{C}^{1}$ with respect to $p$.

b) The discrete Hamiltonian $g: \mathbb{T}^{2} \times \mathbb{R}^{4} \rightarrow \mathbb{R},(x, q) \mapsto g(x, q)$, is continuous with respect to $x$ uniformly in $h$. For all $h \leq h_{0}$, it satisfies $\left(\mathbf{H}_{\mathbf{1}}\right),\left(\mathbf{H}_{\mathbf{2}}\right)$ and $\left(\mathbf{H}_{\mathbf{3}}\right)$.

c) Defining the function $\mathcal{F}: \mathbb{T}^{2} \times \mathbb{R}^{4} \rightarrow \mathbb{R},\left(x, q_{1}, q_{2}, s_{1}, s_{2}\right) \mapsto \mathcal{F}\left(x, q_{1}, q_{2}, s_{1}, s_{2}\right)$ by

$$
-\nu\left(\Delta_{h} U\right)_{i, j}+g\left(x_{i, j},\left[D_{h} U\right]_{i, j}\right)=\mathcal{F}\left(x_{i, j},\left(D_{1}^{c} U\right)_{i, j},\left(D_{2}^{c} U\right)_{i, j},\left(D_{1}^{2} U\right)_{i, j},\left(D_{2}^{2} U\right)_{i, j}\right),
$$

we assume that there exist positive constants $a_{0}, a_{1}$ and $b_{0}$ such that, for $h=1 / N_{h} \leq h_{0}$,

$$
a_{0} \leq-\frac{\partial \mathcal{F}}{\partial s_{i}} \leq a_{1}, \quad \text { and } \quad\left|\frac{\partial \mathcal{F}}{\partial q_{i}}\right| \leq b_{0}
$$

d) There exists a function $g^{\infty}: \mathbb{R}^{4} \rightarrow \mathbb{R}$, such that

- $g^{\infty}(x, 0)=0$,

- for all $q \in \mathbb{R}^{4}$,

$$
\lim _{\epsilon \rightarrow 0} \sup _{x \in \mathbb{T}^{2}}\left|\epsilon g\left(x, \frac{q}{\epsilon}\right)-g^{\infty}(q)\right|=0
$$

- $g^{\infty}$ is nonincreasing with respect to $q_{1}$ and $q_{3}$ and nondecreasing with respect to $q_{2}$ and $q_{4}$.

Example 1 Let $H$ be given by (30) with $\mathcal{A}=\left\{\alpha \in \mathbb{R}^{2},|\alpha| \leq 1\right\}$ and $L(x, \alpha)=L(\alpha)=\frac{1}{\gamma}|\alpha|^{\gamma}$, with $\gamma>1$. It can be seen that

$$
H(x, p)=H(p)= \begin{cases}\frac{\gamma-1}{\gamma}|p|^{\frac{\gamma}{\gamma-1}}, & |p| \leq 1, \\ |p|-\frac{1}{\gamma}, & |p| \geq 1\end{cases}
$$

and that with the Godunov scheme

$$
g\left(x, q_{1}, q_{2}, q_{3}, q_{4}\right)=H\left(\sqrt{\left(q_{1}^{-}\right)^{2}+\left(q_{3}^{-}\right)^{2}+\left(q_{2}^{+}\right)^{2}+\left(q_{4}^{+}\right)^{2}}\right),
$$

Assumption 1 holds with in point d), $g^{\infty}\left(x, q_{1}, q_{2}, q_{3}, q_{4}\right)=\sqrt{\left(q_{1}^{-}\right)^{2}+\left(q_{3}^{-}\right)^{2}+\left(q_{2}^{+}\right)^{2}+\left(q_{4}^{+}\right)^{2}}$. 
Assumption 2 a) The Hamiltonian $H$ is of the form (30) where $\mathcal{A}$ is a compact subset of $\mathbb{R}^{2}$ and $L$ is a $\mathcal{C}^{0}$ function on $\mathbb{T}^{2} \times \mathcal{A}$. The function $H$ is Lipschitz continuous with respect to $x$ uniformly in $p$ and of class $\mathcal{C}^{1}$ with respect to $p$.

b) The discrete Hamiltonian $g$ satisfies point b) in Assumption 1.

c) The discrete Hamiltonian $g$ is of the form

$$
g\left(x, q_{1}, q_{2}, q_{3}, q_{4}\right)=\sup _{\beta \in \mathcal{B}}\left(\sum_{\ell=1}^{4}\left(-a_{\ell}(x, \beta) s_{\ell}+b_{\ell}(x, \beta) q_{\ell}\right)-f(x, \beta)\right),
$$

where

- $\mathcal{B}$ is a compact set,

- $s_{1}=s_{2}=\left(q_{1}-q_{2}\right) / h, s_{3}=s_{4}=\left(q_{3}-q_{4}\right) / h$,

- $a_{1}=a_{2} \geq 0$ and $a_{3}=a_{4} \geq 0$,

- the functions $a_{\ell}, b_{\ell}: \mathbb{T}^{2} \times \mathcal{B} \rightarrow \mathbb{R}$ are continuous with respect to $\beta$ (uniformly w.r.t. $h$ ); $b_{\ell}$ and $\sqrt{\frac{\nu}{2}+a_{\ell}}$ are Lipschitz continuous with respect to $x$ (uniformly w.r.t. $h$ ).

- the function $f: \mathbb{T}^{2} \times \mathcal{B} \rightarrow \mathbb{R}$ is continuous with respect to $\beta$ and Lipschitz continuous with respect to $x$.

- For all $h \leq h_{0},(x, \beta) \in \mathbb{T}^{2} \times \mathcal{B}, \max \left(h b_{1}^{+}(x, \beta)-a_{1}(x, \beta), h b_{2}^{-}(x, \beta)-a_{2}(x, \beta), h b_{3}^{+}(x, \beta)-\right.$ $\left.a_{3}(x, \beta), h b_{4}^{-}(x, \beta)-a_{4}(x, \beta)\right) \leq 0$.

d) The discrete Hamiltonian g satisfies point d) in Assumption 1.

Example 2 We take $H$ as in Example 1. The Lax-Friedrichs scheme with a large enough artificial viscosity parameter $\theta$ satisfies Assumptions 1. It also satisfies Assumptions 2, because

$$
\begin{aligned}
g\left(x, q_{1}, q_{2}, q_{3}, q_{4}\right) & =H\left(x,\left(\frac{q_{1}+q_{2}}{2}, \frac{q_{3}+q_{4}}{2}\right)\right)-\theta\left(q_{1}-q_{2}+q_{3}-q_{4}\right) \\
& =\sup _{\alpha \in \mathcal{A}}\left(\alpha_{1} \frac{q_{1}+q_{2}}{2}+\alpha_{2} \frac{q_{3}+q_{4}}{2}-\theta\left(q_{1}-q_{2}+q_{3}-q_{4}\right)-L(\alpha)\right) .
\end{aligned}
$$

Example 3 We give a simple example where $H$ only depends on $p_{1}$. Let $H$ be given by (30) with $\mathcal{A}=\{(\alpha, 0),|\alpha| \leq 1\}$ and $L(x,(\alpha, 0))=\frac{1}{\theta}|\alpha|^{\theta}$, with $\theta>1$. It can be seen that $H(x, p)=H\left(p_{1}\right)$ with $H\left(p_{1}\right)=\frac{\theta-1}{\theta}\left|p_{1}\right|^{\frac{\theta}{\theta-1}}$ if $\left|p_{1}\right| \leq 1$ and $H\left(p_{1}\right)=\left|p_{1}\right|-\frac{1}{\theta}$ if $\left|p_{1}\right| \geq 1$. Consider the discrete Hamiltonian

$$
g\left(x, q_{1}, q_{2}, q_{3}, q_{4}\right)=\max _{\left|\beta_{1}\right| \leq 1,\left|\beta_{2}\right| \leq 1}\left(-\beta_{1}^{-} q_{1}+\beta_{2}^{+} q_{2}-\frac{1}{\theta}\left|\beta_{1}\right|^{\theta}-\frac{1}{\theta}\left|\beta_{2}\right|^{\theta}\right),
$$

It can be checked that Assumption 2 holds. In particular, $g^{\infty}\left(x, q_{1}, q_{2}, q_{3}, q_{4}\right)=\max \left(q_{2},-q_{1}, q_{2}-\right.$ $\left.q_{1}, 0\right)$. 
Proposition 1 Assume that $H$ and $g$ satisfy Assumption 1 or Assumption 2. Let $V$ be a grid function on $\mathbb{T}_{h}^{2}$ (we agree to write $V$ instead of $V_{h}$ ) and $\rho$ be a nonnegative real number.

For $h \leq h_{0}$, let the grid function $U$ on $\mathbb{T}_{h}^{2}$ be the solution of

$$
\rho U_{i, j}+g\left(x_{i, j},\left[D_{h} U\right]_{i, j}\right)-\nu\left(\Delta_{h} U\right)_{i, j}=V_{i, j},
$$

see Lemma 1.

If Assumption 1 holds and if $\|V\|_{\infty}$ is bounded uniformly w.r.t $h \leq h_{0}$ by a constant $c_{0}$, then there exist two constants $\delta, \delta \in(0,1)$ and $C, C>0$, both depending on $a_{0}, a_{1}, b_{0}, c_{0}$ and on $\|U\|_{\infty}$ but not on $h$ and $\rho$, such that for all $h \leq h_{0}, 0<\rho \leq 1$

$$
\left|U(\xi)-U\left(\xi^{\prime}\right)\right| \leq C\left|\xi-\xi^{\prime}\right|^{\delta}, \quad \forall \xi, \xi^{\prime} \in \mathbb{T}_{h}^{2}
$$

If Assumption 2 holds and if

$$
\|V\|_{\infty}+\left\|D_{h} V\right\|_{\infty} \text { is bounded uniformly w.r.t. } h \leq h_{0} \text { by a constant } c_{0},
$$

there exists a constant $C, C>0$ depending on $\|U\|_{\infty}$ but not on $h$ and $\rho$, such that $\forall h \leq h_{0}$, $0<\rho \leq 1$

$$
\left|U(\xi)-U\left(\xi^{\prime}\right)\right| \leq C\left|\xi-\xi^{\prime}\right|, \quad \forall \xi, \xi^{\prime} \in \mathbb{T}_{h}^{2}
$$

Proof. In the first case, the result is a consequence of a theorem due to Kuo and Trudinger, see formula (3.10) in [9] and also [8], (which makes use of (32)).

In the second case, (38) is a particular case of a discrete Lipschitz estimate recently proved by Krylov with a very clever discrete Bernstein method, see [7], Theorem 2.5 and Remark 4.5.

Remark 2 To cast the discrete quasilinear operator into the setting of Theorem 2.5 in [7], one needs to consider the grid function $W_{i, j}=-U_{i, j}$.

In Proposition 1 the constants $C$ depend on $\left\|U^{\rho}\right\|_{\infty}$. It is possible to improve this result by realizing that the constants actually depend on $\left\|U^{\rho}-U_{0,0}^{\rho}\right\|_{\infty}$ and by showing that this quantity is bounded uniformly w.r.t. $\rho$ and $h$ : the following proposition is due to F. Camilli and C. Marchi, see [3]. We give its proof since [3] is not published yet.

Proposition 2 1. If Assumption 1 holds and if $\|V\|_{\infty}$ is bounded uniformly w.r.t $h \leq h_{0}$ by a constant $c_{0}$, then there exist two constants $\delta, \delta \in(0,1)$ and $C, C>0$ both independent of $h \leq h_{0}$ and $\rho$ such that for all $\rho, 1 \geq \rho>0$, the solution of (35) satisfies

$$
\max _{\xi \neq \xi^{\prime} \in \mathbb{T}_{h}^{2}} \frac{\left|U^{(\rho)}(\xi)-U^{(\rho)}\left(\xi^{\prime}\right)\right|}{\left|\xi-\xi^{\prime}\right|^{\delta}} \leq C .
$$

2. If Assumption 1 and (37) hold, then there exists a constant $C>0$ independent of $h \leq h_{0}$ and $\rho$, such that for all $\rho, 1 \geq \rho>0$, the solution of (35) satisfies

$$
\max _{\xi \neq \xi^{\prime} \in \mathbb{T}_{h}^{2}} \frac{\left|U^{(\rho)}(\xi)-U^{(\rho)}\left(\xi^{\prime}\right)\right|}{\left|\xi-\xi^{\prime}\right|} \leq C .
$$

Proof. We give the proof in the first case only, since the second case is done similarly.

Lemma 1 yields he existence for (35). We also easily obtain a bound on $\left\|\rho U^{(\rho)}\right\|_{\infty}$, namely that

$$
\left\|\rho U^{(\rho)}\right\|_{\infty} \leq \max _{i, j}\left(\left|H\left(x_{i, j}, 0\right)\right|+\left|V_{i, j}\right|\right)
$$


so there exists a positive constant $C_{1}$ independent of $h$ and $\rho$ such that $\left\|\rho U^{(\rho)}\right\|_{\infty} \leq C_{1}$.

Let us have $\rho$ tend to 0 . We set $W^{(\rho)}=U^{(\rho)}-U_{0,0}^{(\rho)}$. Fixing $h$, assume that there exists a sequence $\rho_{k}$ such that $\lim _{k \rightarrow \infty}\left\|W^{\left(\rho_{k}\right)}\right\|_{\infty}=+\infty$. We use the notation $\epsilon_{k}=1 /\left\|W^{\left(\rho_{k}\right)}\right\|_{\infty}$. The grid function $Z^{(k)}=\epsilon_{k} W^{\left(\rho_{k}\right)}$ satisfies

$$
\begin{gathered}
Z_{0,0}^{(k)}=0, \quad\left\|Z^{(k)}\right\|_{\infty}=1, \\
\frac{\rho_{k}}{\epsilon_{k}} Z_{i, j}^{(k)}-\frac{\nu}{\epsilon_{k}}\left(\Delta_{h} Z^{(k)}\right)_{i, j}+g\left(x_{i, j}, \frac{1}{\epsilon_{k}}\left[D_{h} Z^{(k)}\right]_{i, j}\right)+\rho_{k} U_{0,0}^{\left(\rho_{k}\right)}=V_{i, j} .
\end{gathered}
$$

But (43) is equivalent to

$$
\rho_{k} Z_{i, j}^{(k)}-\nu\left(\Delta_{h} Z^{(k)}\right)_{i, j}+\epsilon_{k} g\left(x_{i, j}, \frac{1}{\epsilon_{k}}\left[D_{h} Z^{(k)}\right]_{i, j}\right)+\rho_{k} \epsilon_{k} U_{0,0}^{\left(\rho_{k}\right)}=\epsilon_{k} V_{i, j} .
$$

Note that $-\nu\left(\Delta_{h} Z^{(k)}\right)_{i, j}+\epsilon_{k} g\left(x_{i, j}, \frac{1}{\epsilon_{k}}\left[D_{h} Z^{(k)}\right]_{i, j}\right)=\mathcal{G}\left(x_{i, j},\left(D_{1}^{c} Z\right)_{i, j},\left(D_{2}^{c} Z\right)_{i, j},\left(D_{1}^{2} Z\right)_{i, j},\left(D_{2}^{2} Z\right)_{i, j}\right)$ where $\mathcal{G}\left(x, q_{1}, q_{2}, s_{1}, s_{2}\right)=\epsilon \mathcal{F}\left(x, \frac{q_{1}}{\epsilon}, \frac{q_{2}}{\epsilon}, \frac{s_{1}}{\epsilon}, \frac{s_{2}}{\epsilon}\right)$, so $\mathcal{G}$ also satisfies estimate (32).

From this and (41), we can apply estimate (36) to $Z^{(k)}$ : we get that the grid functions $Z^{(k)}$ are equibounded and equicontinuous. Up to a subsequence, $Z^{(k)}$ converges to $Z$ as $k$ tends to infinity and $Z$ satisfies:

$$
Z_{0,0}=0 \quad \text { and } \quad-\nu\left(\Delta_{h} Z\right)_{i, j}+g^{\infty}\left(x_{i, j},\left[D_{h} Z\right]_{i, j}\right)=0, \quad \forall i, j,
$$

The assumptions made above on $g^{\infty}$ and the discrete maximum principle yield that $Z=0$ which contradicts (42). We have proved that $\left\|U^{(\rho)}-U_{0,0}^{(\rho)}\right\|_{\infty} \leq C$ for a constant $C$ independent of $\rho$, and (39) is a consequence of Proposition 1.

It can be shown by a similar contradiction argument using Ascoli-Arzela theorem that the constant $C$ in the bound $\left\|U^{(\rho)}-U_{0,0}^{(\rho)}\right\|_{\infty} \leq C$ does not depend on $h$, because the unique viscosity solution of $-\nu \Delta z+\sup _{\alpha \in \mathcal{A}} \alpha . \nabla z=0$ with $z(0)=0$ is 0 .

\subsection{Existence for the discrete problem}

Theorem 1 If Assumption 1 is satisfied and if the operator $V$ maps the probability measures to a bounded set of continuous functions on $\mathbb{T}^{2}$ and satisfies $\left(\mathbf{A}_{\mathbf{2}}\right)$, then the discrete problem (23)-(25) has at least a solution, and there exist two constants $\delta, \delta \in(0,1)$ and $C>0$ such that for all $h=1 / N_{h}<h_{0}$,

$$
\|U\|_{\infty}+\max _{\xi \neq \xi^{\prime} \in \mathbb{T}_{h}^{2}} \frac{\left|U(\xi)-U\left(\xi^{\prime}\right)\right|}{\left|\xi-\xi^{\prime}\right|^{\delta}} \leq C
$$

\section{Proof.}

Step 1 We consider a mapping $\Phi: M \in \mathcal{K} \rightarrow U$, where $U$ is part of the solution of the problem: find a grid function $U$ and a scalar $\lambda$ such that

$$
-\nu\left(\Delta_{h} U\right)_{i, j}+g\left(x_{i, j},\left[D_{h} U\right]_{i, j}\right)+\lambda=\left(V_{h}[M]\right)_{i, j},
$$

with $\sum_{i, j} U_{i, j}=0$. Indeed, it can be proved that if $g$ satisfies the assumptions mentioned above, then there exist a unique $\lambda \in \mathbb{R}$ and a unique grid function $U$ satisfying (46). To do it, we pass to the limit in (28) as $\rho \rightarrow 0$. The existence and uniqueness for (28) stems from Lemma 1. We may apply Proposition 2 since $V_{h}[M]$ is bounded uniformly w.r.t. $h$ and $M$. Proposition 2 implies that there exists two constants $C>0$ and $\delta \in(0,1)$ independent of $h, M$ and $\rho$ such 
that (39) holds. This yields that there exists a constant $c(h) \sim h^{\delta-1}$ independent of $M$ and $\rho$ such that

$$
\left\|D_{h} U^{(\rho)}\right\|_{\infty} \leq c(h)
$$

Up to the extraction of a subsequence we may say that as $\rho$ tends to $0, U^{(\rho)}-h^{2} \sum_{i, j} U_{i, j}^{(\rho)}$ converges to a grid function $U$ such that $\sum_{i, j} U_{i, j}=0$, and that $\rho h^{2} \sum_{i, j} U_{i, j}^{(\rho)}$ converges to $\lambda \in \mathbb{R}$. It is an easy matter to check that $(U, \lambda)$ satisfies (46) and that the bounds (39) and (47) hold for $U$.

Uniqueness for $\lambda$ stems from the following comparison principle: if $U$ is a subsolution of (46) with $\lambda=\lambda_{1}$ and $W$ is a supersolution of (46) with $\lambda=\lambda_{2}$, then $\lambda_{2} \leq \lambda_{1}$. Uniqueness for $U$ is obtained by repeatedly applying the discrete maximum principle, from the monotonicity of $g$. We have defined the map $\Phi: M \in \mathcal{K} \rightarrow U$, where $(U, \lambda)$ solve (46) and $\sum_{i, j} U_{i, j}=0$.

Step 2: continuity of $\Phi$ Consider a sequence of grid functions $M^{(k)}$ in $\mathcal{K}$ which tends to $M \in \mathcal{K}$ as $k$ tends to infinity. From the assumptions on $V$ and $V_{h}, V_{h}\left[M^{(k)}\right]$ converges to $V_{h}[M]$. Consider $\lambda$ and $U$ solution of (46) and call $\lambda^{(k)}, U^{(k)}$ a solution of (46) with $M=M^{(k)}$. From the estimates above, the sequences $\left(\lambda^{k}\right)_{k}$ and $\left(\left\|U^{(k)}\right\|_{\infty}\right)_{k}$ are bounded. One can extract a subsequence $k^{\prime}$ such that $\lambda^{(k)}$ tends to $\widetilde{\lambda}$ and $U^{\left(k^{\prime}\right)}$ tends to $\widetilde{U}$ and that

$$
-\nu\left(\Delta_{h} \widetilde{U}\right)_{i, j}+g\left(x_{i, j},\left[D_{h} \widetilde{U}\right]_{i, j}\right)+\widetilde{\lambda}=\left(V_{h}[M]\right)_{i, j},
$$

with $\sum_{i, j} \widetilde{U}_{i, j}=0$.

Uniqueness for (46) implies that $\tilde{\lambda}=\lambda$ and $\widetilde{U}=U$. The whole sequences $\left(\lambda^{(k)}\right)_{k},\left(U^{(k)}\right)_{k}$ therefore tend to $\lambda, U$.

We have proved that the map $\Phi$ is continuous.

Step 3 For $M \in \mathcal{K}$ and $U=\Phi(M)$, consider the following linear problem: find $\widetilde{M}$ such that

$$
\mu \widetilde{M}_{i, j}-\nu\left(\Delta_{h} \widetilde{M}\right)_{i, j}-\mathcal{B}_{i, j}(U, \widetilde{M})=\mu M_{i, j}
$$

where $\mu$ is a sufficiently large positive number which will be chosen later. This linear problem may be written

$$
\mu \widetilde{M}+A \widetilde{M}=\mu M
$$

where $A$ is a linear operator depending on $U$.

The assumptions of the monotonicity of $g$ imply that $\frac{\partial g}{\partial q_{1}} \leq 0, \frac{\partial g}{\partial q_{2}} \geq 0, \frac{\partial g}{\partial q_{3}} \leq 0$ and $\frac{\partial g}{\partial q_{4}} \geq 0$. This yields that the matrix corresponding to $A$ has positive diagonal entries and nonpositive off-diagonal entries. Furthermore, since $g$ is $\mathcal{C}^{1}$, (47) implies that there exists a constant $C$ independent of $M$ (but possibly on $h$ ) such that for all $i, j, 0 \leq i, j \leq N_{h}$, and for all $\ell=1,2,3,4$,

$$
\left|\frac{\partial g}{\partial q_{\ell}}\left(x_{i, j},\left[D_{h} U\right]_{i, j}\right)\right| \leq C .
$$

From this, we see that for $\mu$ large enough depending possibly on $h$ but not on $M$, the matrix corresponding to $\mu I d+A$ is a M-matrix, and is therefore invertible. The system of linear equations (48) has a unique solution $\widetilde{M}$ and $\widetilde{M}$ is nonnegative since $M$ is nonnegative.

We are left with proving that $h^{2}(\widetilde{M}, 1)_{2}=h^{2}(M, 1)_{2}=1$. For two grid functions $W$ and $Z$, let 
us compute $\left(A^{n} W, Z\right)_{2}$ : discrete integrations by part lead to

$$
\begin{aligned}
& (A W, Z)_{2}= \\
& \frac{\nu}{h^{2}} \sum_{i, j}\left(\left(W_{i, j}-W_{i-1, j}\right)\left(Z_{i, j}-Z_{i-1, j}\right)+\left(W_{i, j}-W_{i, j-1}\right)\left(Z_{i, j}-Z_{i, j-1}\right)\right) \\
& +\left(\begin{array}{c}
\sum_{i, j}\left(D_{1}^{+} Z\right)_{i, j} W_{i, j} \frac{\partial g}{\partial q_{1}}\left(x_{i, j},\left[D_{h} U\right]_{i, j}\right) \\
+\sum_{i, j}\left(D_{1}^{+} Z\right)_{i-1, j} W_{i, j} \frac{\partial g}{\partial q_{2}}\left(x_{i, j},\left[D_{h} U\right]_{i, j}\right)
\end{array}\right)+\left(\begin{array}{l}
\sum_{i, j}\left(D_{2}^{+} Z\right)_{i, j} W_{i, j} \frac{\partial g}{\partial q_{3}}\left(x_{i, j},\left[D_{h} U\right]_{i, j}\right) \\
+\sum_{i, j}\left(D_{2}^{+} Z\right)_{i, j-1} W_{i, j} \frac{\partial g}{\partial q_{4}}\left(x_{i, j},\left[D_{h} U\right]_{i, j}\right)
\end{array}\right) \\
& (A W, Z)_{2}=\nu \sum_{i, j}\left(D_{1}^{+} W\right)_{i, j}\left(D_{1}^{+} Z\right)_{i, j}+\nu \sum_{i, j}\left(D_{2}^{+} W\right)_{i, j}\left(D_{2}^{+} Z\right)_{i, j} \\
& +\sum_{i, j} W_{i, j}\left[D_{h} Z\right]_{i, j} \cdot \nabla_{q} g\left(x_{i, j},\left[D_{h} U\right]_{i, j}\right) .
\end{aligned}
$$

It is easy to check that for all grid functions $W,(A W, 1)_{2}=0$. Therefore, taking the inner product of (49) with the function $Z=1$, we obtain that $h^{2}(\widetilde{M}, 1)_{2}=h^{2}(M, 1)_{2}=1$, so $\widetilde{M} \in \mathcal{K}$. We call $\chi: \mathcal{K} \mapsto \mathcal{K}$ the mapping defined by $\chi: M \rightarrow \widetilde{M}$.

Step 4: existence of a fixed point of $\chi$ From the boundedness and continuity of the mapping $\Phi$, and from the fact that $g$ is $\mathcal{C}^{1}$ in the variable $q$, we obtain that $\chi$ is continuous. Therefore, we can apply Brouwer fixed point theorem and obtain that $\chi$ has a fixed point.

We obtain a better result under Assumption 2 and a stronger assumptions on $V$ :

Theorem 2 If Assumption 2 holds and if $V$ satisfies $\left(\mathbf{A}_{\mathbf{1}}\right)$ and $\left(\mathbf{A}_{\mathbf{2}}\right)$, then the discrete problem (23)-(25) has at least a solution, and there exists a constant $C>0$ such that for all $h=1 / N_{h}<$ $h_{0}$,

$$
\|U\|_{\infty}+\max _{\xi \neq \xi^{\prime} \in \mathbb{T}_{h}^{2}} \frac{\left|U(\xi)-U\left(\xi^{\prime}\right)\right|}{\left|\xi-\xi^{\prime}\right|} \leq C
$$

Proof. Similar to that of Theorem 1, using now the second part of Proposition 2.

Since the discrete problem is finite-dimensional, existence for (23)-(25) can be proved without a bound on $U$ uniform with respect to $h$. Different assumptions on the structure of $g$ can be made; for example:

Theorem 3 Assume that

- $g$ satisfies $\left(\mathbf{H}_{\mathbf{1}}\right),\left(\mathbf{H}_{\mathbf{2}}\right)$ and $\left(\mathbf{H}_{\mathbf{3}}\right)$,

- there exist two positive constants $\alpha>0$ and $\gamma>1$ and a nonnegative constant $C$ such that

$$
g\left(x, q_{1}, q_{2}, q_{3}, q_{4}\right) \geq \alpha\left(\left(q_{1}^{-}\right)^{2}+\left(q_{2}^{+}\right)^{2}+\left(q_{3}^{-}\right)^{2}+\left(q_{4}^{+}\right)^{2}\right)^{\gamma / 2}-C, \quad \forall x \in \mathbb{T}^{2} .
$$

- $V$ maps the probability measures to a bounded set of continuous functions on $\mathbb{T}^{2}$ and satisfies $\left(\mathbf{A}_{\mathbf{1}}\right)$ and $\left(\mathbf{A}_{\mathbf{2}}\right)$.

The discrete problem (23)-(25) has at least a solution 
Proof. The proof follows the same steps as that of Theorem 1. Only the first step of the proof is modified as follows:

existence and uniqueness for (28) follows from Lemma 1. We also easily obtain a bound on $\left\|\rho U^{(\rho)}\right\|_{\infty}$, namely that

$$
\left\|\rho U^{(\rho)}\right\|_{\infty} \leq \max _{i, j}\left|H\left(x_{i, j}, 0\right)+\left(V_{h}[M]\right)_{i, j}\right|,
$$

so there exists a positive constant $C_{1}$ independent of $M$ and $\rho$ such that $\left\|\rho U^{(\rho)}\right\|_{\infty} \leq C_{1}$. From this, we deduce that there exists a positive constant $C_{2}$ independent of $M$ and $\rho$ such that

$$
g\left(x_{i, j},\left[D_{h} U^{(\rho)}\right]_{i, j}\right)-\nu\left(\Delta_{h} U^{(\rho)}\right)_{i, j} \leq C_{2}, \quad \forall i, j .
$$

Using (54), we see that

$$
\begin{aligned}
& g\left(x_{i, j},\left[D_{h} W\right]_{i, j}\right)-\nu\left(\Delta_{h} W\right)_{i, j} \\
\geq & \alpha\left(\left(\left(D_{1}^{+} W\right)_{i, j}^{-}\right)^{2}+\left(\left(D_{1}^{+} W\right)_{i-1, j}^{+}\right)^{2}+\left(\left(D_{2}^{+} W\right)_{i, j}^{-}\right)^{2}+\left(\left(D_{2}^{+} W\right)_{i, j-1}^{+}\right)^{2}\right)^{\gamma / 2}-C \\
& -\frac{\nu}{h}\left(\left(D_{1}^{+} W\right)_{i, j}^{+}+\left(D_{1}^{+} W\right)_{i-1, j}^{-}+\left(D_{2}^{+} W\right)_{i, j}^{+}+\left(D_{2}^{+} W\right)_{i, j-1}^{-}\right) .
\end{aligned}
$$

Calling $P_{\rho}=\left\|D_{h} U^{(\rho)}\right\|_{\infty}=\max _{i, j} \max \left(\left|\left(D_{1}^{+} U^{(\rho)}\right)_{i, j}\right|,\left|\left(D_{2}^{+} U^{(\rho)}\right)_{i, j}\right|\right)$, we deduce from (56) and the previous estimate that there exists a constant $C_{3}$ independent of $M$ and $\rho$ such that

$$
\alpha P_{\rho}^{\gamma}-4 \frac{\nu}{h} P_{\rho} \leq C_{3}
$$

This yields (47) for a constant $c(h)$ independent of $M$ and $\rho$. Up to the extraction of a subsequence we may say that as $\rho$ tends to $0, U^{(\rho)}-h^{2} \sum_{i, j} U_{i, j}^{(\rho)}$ converges to a grid function $U$ such that $\sum_{i, j} U_{i, j}=0$, and that $\rho h^{2} \sum_{i, j} U_{i, j}^{(\rho)}$ converges to $\lambda \in \mathbb{R}$. The limits $U$ and $\lambda$ satisfy (46). Uniqueness for (46) is proved as above, so $\Phi$ is well defined.

Remark 3 In Theorem 3, we were not able to obtain an estimate on U uniform w.r.t. h.

Remark 4 Note that the assumption $\left(\mathbf{A}_{\mathbf{2}}\right)$ can be relaxed in the discrete case. Indeed, Theorem 1 holds if we replace $\left(\mathbf{A}_{2}\right)$ with the assumption that $V_{h}$ is a continuous map from $\mathcal{K}$ defined in (27) to grid functions bounded by a constant independent of $h$. Theorem 3 holds if $V_{h}$ is a continuous map from $\mathcal{K}$ to grid functions.

These observations lead to existence results when $V_{h}$ is a local operator, see $\S 3.4$.

\subsection{Uniqueness}

Proposition 3 If $g$ satisfies $\left(\mathbf{H}_{\mathbf{1}}\right)-\left(\mathbf{H}_{\mathbf{4}}\right)$, and if the operator $V_{h}$ is strictly monotone, i.e.

$$
\left(V_{h}[M]-V_{h}[\widetilde{M}], M-\widetilde{M}\right)_{2} \leq 0 \Rightarrow M=\widetilde{M}
$$

then (23)-(25) has at most a solution.

Proof. Let $(U, M, \lambda)$ and $\widetilde{U}, \widetilde{M}, \widetilde{\lambda}$ be two solutions of (23)-(25). We have

$$
-\nu\left(\Delta_{h}(U-\widetilde{U})\right)_{i, j}+g\left(x_{i, j},\left[D_{h} U\right]_{i, j}\right)-g\left(x_{i, j},\left[D_{h} \widetilde{U}\right]_{i, j}\right)+\lambda-\widetilde{\lambda}=\left(V_{h}[M]-V_{h}[\widetilde{M}]\right)_{i, j} .
$$


Take a grid function such that $\sum_{i, j} Z_{i, j}=0$. Multiplying by $Z_{i, j}$ and summing over all $i, j$ leads to

$$
-\nu\left(\Delta_{h}(U-\widetilde{U}), Z\right)_{2}+\sum_{i, j}\left(g\left(x_{i, j},\left[D_{h} U\right]_{i, j}\right)-g\left(x_{i, j},\left[D_{h} \widetilde{U}\right]_{i, j}\right)\right) Z_{i, j}=\left(V_{h}[M]-V_{h}[\widetilde{M}], Z\right)_{2} .
$$

On the other hand, multiplying the second equation in (23) by $-W_{i, j}$ and summing over all $i, j$ leads to

$$
\nu\left(M, \Delta_{h} W\right)_{2}-\sum_{i, j} M_{i, j}\left[D_{h} W\right]_{i, j} \cdot \nabla_{q} g\left(x_{i, j},\left[D_{h} U\right]_{i, j}\right)=0 .
$$

From this and the similar equation satisfied by $\widetilde{M}$, we obtain

$$
\begin{aligned}
0= & \nu\left((M-\widetilde{M}), \Delta_{h} W\right)_{2} \\
& -\sum_{i, j} M_{i, j}\left[D_{h} W\right]_{i, j} \cdot \nabla_{q} g\left(x_{i, j},\left[D_{h} U\right]_{i, j}\right)+\sum_{i, j} \widetilde{M}_{i, j}\left[D_{h} W\right]_{i, j} \cdot \nabla_{q} g\left(x_{i, j},\left[D_{h} \widetilde{U}\right]_{i, j}\right) .
\end{aligned}
$$

Taking $Z=M-\widetilde{M}$ in (58), $W=U-\widetilde{U}$ in (60) and adding the resulting equations leads to

$$
\begin{array}{r}
\sum_{i, j} M_{i, j}\left(g\left(x_{i, j},\left[D_{h} \widetilde{U}\right]_{i, j}\right)-g\left(x_{i, j},\left[D_{h} U\right]_{i, j}\right)-D_{h}(\widetilde{U}-U)_{i, j} \cdot \nabla_{q} g\left(x_{i, j},\left[D_{h} U\right]_{i, j}\right)\right) \\
+\sum_{i, j} \widetilde{M}_{i, j}\left(g\left(x_{i, j},\left[D_{h} U\right]_{i, j}\right)-g\left(x_{i, j},\left[D_{h} \widetilde{U}\right]_{i, j}\right)-D_{h}(U-\widetilde{U})_{i, j} \cdot \nabla_{q} g\left(x_{i, j},\left[D_{h} \widetilde{U}\right]_{i, j}\right)\right) \\
+\left(V_{h}[M]-V_{h}[\widetilde{M}], M-\widetilde{M}\right)_{2}=0 .
\end{array}
$$

From the convexity of $g$ and the monotonicity assumption of $F$, the three terms in (61) must vanish. The strong monotonicity of $V_{h}$ implies that $M=\widetilde{M}$. A comparison argument similar to that used in the first step of the proof of Theorem 1 yields that $\lambda=\widetilde{\lambda}$ and that $U=\widetilde{U}$.

\subsection{The case when $V$ is a local operator}

We now aim at relaxing the assumptions on $V$ : we now assume that $V$ is a local operator, i.e. $V[m](x)=F(m(x), x)$, where $F$ is a bounded and $\mathcal{C}^{0}$ function defined on $\mathbb{R} \times \mathbb{T}^{2}$.

\subsubsection{Existence}

From Remark 4, we have the analogues of Theorems 1 and 3:

Proposition 4 Take $V$ as above. If assumption 1 holds, then the discrete problem (23)-(25) has at least a solution, and there exist two constants $\delta, \delta \in(0,1)$ and $C>0$ such that (45) is satisfied for all $h=1 / N_{h}<h_{0}$.

Proposition 5 If $g$ satisfies the same assumptions as in Theorem 3 and if $V[m](x)=F(m(x), x)$ with $F$ is $\mathcal{C}^{0}$ function defined on $\mathbb{R} \times \mathbb{T}^{2}$, then the problem (23)-(25) has at least a solution.

Remark 5 In Proposition 5, we do not have a bound on $U$ uniform w.r.t. $h$.

\subsubsection{Uniqueness}

We have the corollary of Proposition 3:

Corollary 1 If $g$ satisfies assumptions $\left(\mathbf{H}_{\mathbf{1}}\right)-\left(\mathbf{H}_{\mathbf{4}}\right)$ and if $F$ is strictly monotone, i.e.

$$
(F(m, x)-F(\tilde{m}, x))(m-\tilde{m}) \leq 0 \Rightarrow m=\tilde{m},
$$

then (23)-(25) has at most a solution. 


\subsection{A convergence result}

It is possible to prove several convergence results. We give the simplest one as an example.

Theorem 4 We make the same assumptions as in Theorem 1 and we suppose furthermore that $\left(\mathbf{H}_{\mathbf{4}}\right)-\left(\mathbf{H}_{\mathbf{5}}\right)$ hold and that there exist real numbers $c, s, c>0, s>0$, such that for all $h<1$, for all grid functions $M$ and $\widetilde{M}$,

$$
h^{2}\left(V_{h}[M]-V_{h}[\widetilde{M}], M-\widetilde{M}\right)_{2} \geq c\left\|V_{h}[M]-V_{h}[\widetilde{M}]\right\|_{\infty}^{s} .
$$

Assume that (1)-(3) has a unique solution such that $u$ and $m$ belong to $\mathcal{C}^{\ell, \delta_{0}}\left(\mathbb{T}^{2}\right) \cap \mathcal{C}^{2}\left(\mathbb{T}^{2}\right)$, see (22). Calling $\left(U, M, \lambda_{h}\right)$ the solution of the discrete problem (23)-(25), we have

$$
\lim _{h \rightarrow 0} \sup _{i, j}\left|u\left(x_{i, j}\right)-U_{i, j}\right|=0, \quad \lim _{h \rightarrow 0}\left|\lambda-\lambda_{h}\right|=0 .
$$

Proof. We call $\widetilde{U}$ and $\widetilde{M}$ the grid functions such that $\widetilde{U}_{i, j}=h^{-2} \int_{\left|x-x_{i, j}\right|_{\infty}<h / 2} u d x$ and $\widetilde{M}_{i, j}=$ $h^{-2} \int_{\left|x-x_{i, j}\right|_{\infty}<h / 2} m d x$. From the consistency assumptions, we have that

$$
\left\{\begin{aligned}
-\nu\left(\Delta_{h} \widetilde{U}\right)_{i, j}+g\left(x_{i, j},\left[D_{h} \widetilde{U}\right]_{i, j}\right)+\lambda & =\left(V_{h}[\widetilde{M}]\right)_{i, j}+o(1), \\
-\nu\left(\Delta_{h} \widetilde{M}\right)_{i, j}-\mathcal{B}_{i, j}(\widetilde{U}, \widetilde{M}) & =o(1), \\
\widetilde{M}_{i, j} & \geq 0 \\
h^{2} \sum_{i, j} \widetilde{M}_{i, j}=1, \quad \text { and } \sum_{i, j} \widetilde{U}_{i, j} & =0
\end{aligned}\right.
$$

where $o(1)$ means a grid function whose maximum norm tends to 0 as $h$ tends to 0 .

On the other hand, from Theorem $1,\|U\|_{\infty}$ is bounded by a constant.

We therefore obtain an equation close to (61):

$$
\begin{aligned}
o\left(h^{-2}\right)= & \left(V_{h}[M]-V_{h}[\widetilde{M}], M-\widetilde{M}\right)_{2} \\
& +\sum_{i, j} M_{i, j}\left(g\left(x_{i, j},\left[D_{h} \widetilde{U}\right]_{i, j}\right)-g\left(x_{i, j},\left[D_{h} U\right]_{i, j}\right)-D_{h}(\widetilde{U}-U)_{i, j} \cdot \nabla_{q} g\left(x_{i, j},\left[D_{h} U\right]_{i, j}\right)\right) \\
& +\sum_{i, j} \widetilde{M}_{i, j}\left(g\left(x_{i, j},\left[D_{h} U\right]_{i, j}\right)-g\left(x_{i, j},\left[D_{h} \widetilde{U}\right]_{i, j}\right)-D_{h}(U-\widetilde{U})_{i, j} \cdot \nabla_{q} g\left(x_{i, j},\left[D_{h} \widetilde{U}\right]_{i, j}\right)\right) .
\end{aligned}
$$

From (64), the convexity of $g$ and (62), we obtain that $\lim _{h \rightarrow 0}\left\|V_{h}[M]-V_{h}[\widetilde{M}]\right\|_{\infty}=0$. Thus,

$$
\left\{\begin{array}{l}
-\nu\left(\Delta_{h} \widetilde{U}\right)_{i, j}+g\left(x_{i, j},\left[D_{h} \widetilde{U}\right]_{i, j}\right)+\lambda=\left(V_{h}[M]\right)_{i, j}+o(1) \\
-\nu\left(\Delta_{h} U\right)_{i, j}+g\left(x_{i, j},\left[D_{h} U\right]_{i, j}\right)+\lambda_{h}=\left(V_{h}[M]\right)_{i, j}
\end{array}\right.
$$

The maximum principle at the maximum of $\widetilde{U}-U$ yields that $\lambda-\lambda_{h} \leq o(1)$. The same argument at the maximum of $U-\widetilde{U}$ yields $\lambda_{h}-\lambda \leq o(1)$. Therefore, $\lim _{h \rightarrow 0}\left|\lambda-\lambda_{h}\right|=0$.

We know that the family of grid functions $u_{h}$ is equibounded and equicontinuous. There exists a function $u^{\prime}$ and a subsequence $u_{h_{n}}=\left(U_{i, j}^{(n)}\right)_{n} \in \mathbb{N}$ such that $\lim _{h \rightarrow 0} \sup _{i, j}\left|u^{\prime}\left(x_{i, j}\right)-U_{i, j}^{(n)}\right|=0$. The function $u^{\prime}$ is a viscosity solution of (1) and is such that $\int_{\mathbb{T}^{2}} u^{\prime} d x=0$. This implies that $u=u^{\prime}$. Therefore, the whole sequence $u_{h}$ converges to $u$. 


\section{Approximation of the evolution system (8)-(11)}

Let $N_{T}$ be a positive integer and $\Delta t=T / N_{T}, t_{n}=n \Delta t, n=0, \ldots, N_{T}$. The values of $u$ and $m$ at $\left(x_{i, j}, t_{n}\right)$ are respectively approximated by $U_{i, j}^{n}$ and $M_{i, j}^{n}$. Given $M^{0} \in \mathcal{K}$ (the compact set $\mathcal{K}$ is defined in (27) and $U^{0}$, the discrete problem is to look for $\left(U^{n}, M^{n}\right), n=1, \ldots, N_{T}$, such that

$$
\begin{aligned}
& \forall n, i, j: \\
& 0 \leq n<N_{T}, \\
& 0 \leq i, j<N_{h},
\end{aligned} \quad\left\{\begin{aligned}
\frac{U_{i, j}^{n+1}-U_{i, j}}{\Delta t}-\nu\left(\Delta_{h} U^{n+1}\right)_{i, j}+g\left(x_{i, j},\left[D_{h} U^{n+1}\right]_{i, j}\right) & =\left(V_{h}\left[M^{n+1}\right]\right)_{i, j}, \\
\frac{M_{i, j}^{n+1}-M_{i, j}}{\Delta t}-\nu\left(\Delta_{h} M^{n+1}\right)_{i, j}-\mathcal{B}_{i, j}\left(U^{n+1}, M^{n+1}\right) & =0 \\
M_{i, j}^{n+1} \geq 0 & \geq
\end{aligned}\right.
$$

with the notations introduced above (in particular $\mathcal{B}_{i, j}$ is defined in (20)), and

$$
h^{2} \sum_{i, j} M_{i, j}^{n+1}=1, \quad \text { for } n=0, \ldots N_{T}-1 .
$$

\subsection{The main theorem on existence}

Theorem 5 Assume that

- $g$ satisfies $\left(\mathbf{H}_{\mathbf{1}}\right)-\left(\mathbf{H}_{\mathbf{3}}\right)$ and there exists a constant $C$ such that

$$
\mid \frac{\partial g}{\partial x}\left(x,\left(q_{1}, q_{2}, q_{3}, q_{4}\right) \mid \leq C\left(1+\left|q_{1}\right|+\left|q_{2}\right|+\left|q_{3}\right|+\left|q_{4}\right|\right), \quad \forall x \in \mathbb{T}^{2}, \quad \forall q_{1}, q_{2}, q_{3}, q_{4} .\right.
$$

- $V$ satisfies $\left(\mathbf{A}_{\mathbf{1}}\right)$ and $\left(\mathbf{A}_{\mathbf{2}}\right)$.

If $M^{0} \in \mathcal{K}$, then (65) (66) has a solution. If there exists a constant $C$ independent of $h$ such that $\left\|D_{h} U^{0}\right\|_{\infty} \leq C$, then for all $n,\left\|D_{h} U^{n}\right\|_{\infty} \leq c$. for a constant $c$ independent of $h$ and $\delta t$.

Proof. The strategy of the proof is similar to that used for Theorem 1. We are going to construct a continuous mapping $\chi: \mathcal{K}^{N_{T}} \rightarrow \mathcal{K}^{N_{T}}$ and use Brouwer fixed point theorem. We proceed in several steps:

Step 1: a mapping $M \rightarrow U$ Given $\left(U_{i, j}^{0}\right)_{0 \leq i, j<N_{h}}$, Consider the map $\Phi:\left(M^{n}\right)_{1 \leq n \leq N_{T}} \in$ $\mathcal{K}^{N_{T}} \rightarrow\left(U^{n}\right)_{1 \leq n \leq N_{T}}$, solution of the first equation in (65), i.e.

$$
\frac{U_{i, j}^{n+1}-U_{i, j}^{n}}{\Delta t}-\nu\left(\Delta_{h} U^{n+1}\right)_{i, j}+g\left(x_{i, j},\left[D_{h} U^{n+1}\right]_{i, j}\right)=\left(V_{h}\left[M^{n+1}\right]\right)_{i, j},
$$

for $n=0, \ldots N_{T}-1$ and $0 \leq i, j<N_{h}$. The existence and uniqueness of $U^{n+1}, n=0, \ldots, N_{T}-$ 1 is obtained by induction: at each step, we use Lemma 1 with $\rho=\frac{1}{\Delta t}$ and $V_{i, j}=\frac{U_{i, j}^{n}}{\Delta t}+$ $\left(V_{h}\left[M^{n+1}\right]\right)_{i, j}$

Step 2: boundedness and continuity of $\Phi$ Looking at the proof of Lemma 1, we see that

$$
\left\|U^{n+1}\right\|_{\infty} \leq \max _{(i, j)}\left|\Delta t\left(H\left(x_{i, j}, 0\right)-\left(V_{h}\left[M^{n+1}\right]\right)_{i, j}\right)-U_{i, j}^{n}\right|,
$$

which implies, from the uniform boundedness assumption on $V$ and of $H(\cdot, 0)$, that there exists a constant $C$ depending on $\| U^{0}$ but independent of $\left(M^{n}\right)$ such that $\left\|U^{n}\right\|_{\infty} \leq C(1+T)$. Therefore, $\Phi$ maps $\mathcal{K}^{N_{T}}$ to a bounded subset of $\left(\mathbb{R}^{N_{h}^{2}}\right)^{N_{T}}$. Moreover, by using the assumption on the continuity of $V$ and well known results on continuous dependence on the data for monotone schemes (see e.g. [4]), we see that the mapping $\Phi$ is continuous from $\mathcal{K}^{N_{T}}$ to $\left(\mathbb{R}^{N_{h}^{2}}\right)^{N_{T}}$. 
Step 3: discrete Lipschitz continuity estimates on $\Phi\left(\left(M^{n}\right)_{n=1, \ldots, N_{T}}\right)$ We proceed by proving discrete Lipschitz continuity estimates on $U^{n+1}$ given by (68). The solution of (68) is noted

$$
U^{n+1}=\Psi\left(U^{n}, M^{n+1}\right)
$$

Standard arguments on monotone schemes yield that for all $M \in \mathcal{K}, U, W \in \mathbb{R}^{N_{h}^{2}}$,

$$
\begin{array}{cc}
\left\|(\Psi(U, M)-\Psi(W, M))^{+}\right\|_{\infty} & \leq\left\|(U-W)^{+}\right\|_{\infty} \\
\|\Psi(U, M)-\Psi(W, M)\|_{\infty} & \leq\|U-W\|_{\infty} .
\end{array}
$$

For $(\ell, m) \in \mathbb{Z}^{2}$, call $\tau_{\ell, m} U$ the discrete function defined by

$$
\left(\tau_{\ell, m} U\right)_{i, j}=U_{\ell+i, m+j} .
$$

It is a simple matter to check that

$$
\begin{aligned}
& \frac{\left(\tau_{\ell, m} U\right)_{i, j}^{n+1}-\left(\tau_{\ell, m} U\right)_{i, j}^{n}}{\Delta t}-\nu\left(\Delta_{h}\left(\tau_{\ell, m} U^{n+1}\right)\right)_{i, j}+g\left(x_{i, j},\left[D_{h}\left(\tau_{\ell, m} U^{n+1}\right)\right]_{i, j}\right) \\
= & \left(V_{h}\left[M^{n+1}\right]\right)_{i, j}+\left(V_{h}\left[M^{n+1}\right]\right)_{i+\ell, j+m}-\left(V_{h}\left[M^{n+1}\right]\right)_{i, j} \\
& -g\left(x_{i+\ell, j+m},\left[D_{h}\left(\tau_{\ell, m} U^{n+1}\right)\right]_{i, j}\right)+g\left(x_{i, j},\left[D_{h}\left(\tau_{\ell, m} U^{n+1}\right)\right]_{i, j}\right),
\end{aligned}
$$

and therefore

$$
\begin{aligned}
\tau_{\ell, m} U^{n+1} & =\Psi\left(\tau_{\ell, m} U^{n}+\Delta t E, M^{n+1}\right), \\
E_{i, j} & =\left(\begin{array}{c}
\left(V_{h}\left[M^{n+1}\right]\right)_{i+\ell, j+m}-\left(V_{h}\left[M^{n+1}\right]\right)_{i, j} \\
-g\left(x_{i+\ell, j+m},\left[D_{h}\left(\tau_{\ell, m} U^{n+1}\right)\right]_{i, j}\right)+g\left(x_{i, j},\left[D_{h}\left(\tau_{\ell, m} U^{n+1}\right)\right]_{i, j}\right)
\end{array}\right) .
\end{aligned}
$$

From the assumptions on $V$ and on $g$ (in particular (67)), there exists a constant $C$ (independent of $n,\left(M^{n}\right), h$ and $\left.\Delta t\right)$ such that $\|E\|_{\infty} \leq C\left(1+\left\|D_{h} U^{n+1}\right\|_{\infty}\right) h \sqrt{\ell^{2}+m^{2}}$. We conclude from (70) that

$$
\left\|\tau_{\ell, m} U^{n+1}-U^{n+1}\right\|_{\infty} \leq\left\|\tau_{\ell, m} U^{n}-U^{n}\right\|_{\infty}+C h \Delta t \sqrt{\ell^{2}+m^{2}}\left(1+\left\|D_{h} U^{n+1}\right\|_{\infty}\right) .
$$

Thanks to $(71)$,

$$
(1-C \Delta t)\left\|D_{h} U^{n+1}\right\|_{\infty} \leq\left\|D_{h} U^{n}\right\|_{\infty}+C \Delta t .
$$

A discrete version of Gronwall's lemma yields that there exists a constant $L$ which only depends on $C, T$ and the initial condition $\left\|D_{h} U^{0}\right\|_{\infty}$ such that for all $n, 1 \leq n \leq N_{T}$,

$$
\left\|D_{h} U^{n+1}\right\|_{\infty} \leq L
$$

which is a discrete Lipschitz continuity estimate, uniform with respect to $\left(M^{n}\right)_{1 \leq n \leq N_{T}}$.

Step 4: a fixed point problem for $\left(M^{n}\right)_{1 \leq n \leq N_{T}} \quad$ For $\left(M^{n}\right)_{1 \leq n \leq N_{T}} \in \mathcal{K}^{N_{T}}$ and $\left(U^{n}\right)_{1 \leq n \leq N_{T}}=$ $\Phi\left(\left(M^{n}\right)_{1 \leq n \leq N_{T}}\right)$ and a positive real number $\mu$, consider the following linear problem: find $\left(\widetilde{M}^{n}\right)_{1 \leq n \leq N_{T}}$ such that

$$
\frac{\widetilde{M}_{i, j}^{n+1}-\widetilde{M}_{i, j}^{n}}{\Delta t}+\mu \widetilde{M}_{i, j}^{n+1}-\nu\left(\Delta_{h} \widetilde{M}^{n+1}\right)_{i, j}-\mathcal{B}_{i, j}\left(U^{n+1}, \widetilde{M}^{n}\right)=\mu M_{i, j}^{n+1}
$$

with the initial condition $\widetilde{M^{0}}=M^{0}$, with $h^{2} \sum_{i, j} M_{i, j}^{0}=1$ and $M^{0} \geq 0$.

We are going to prove first that for $\mu$ large enough, (73) has a unique solution $\left(\widetilde{M}^{n}\right)_{1 \leq n \leq N_{T}} \in$ $\mathcal{K}^{N_{T}}$, then that the mapping $\left(M^{n}\right)_{1 \leq n \leq N_{T}} \rightarrow\left(\widetilde{M}^{n}\right)_{1 \leq n \leq N_{T}}$ has a fixed point. Existence for (65) (66) will then be proved. 
Step 5: existence for (73) Clearly (73) is a discrete version of a linear parabolic initial value problem. It can be written

$$
\widetilde{M}^{n+1}+\Delta t\left(\mu \widetilde{M}^{n+1}+A^{n+1} \widetilde{M}^{n+1}\right)=\widetilde{M}^{n}+\mu \Delta t M^{n+1},
$$

where $A^{n+1}$ is a linear operator depending on $U^{n+1}$.

As in the proof of Theorem 1, The assumptions on the monotonicity of $g$ imply that the matrix corresponding to $I d+\Delta t A^{n+1}$ has positive diagonal entries and nonpositive off-diagonal entries. Furthermore, since $g$ is $\mathcal{C}^{1},(72)$ implies that there exists a constant $C$ depending only of $\left\|D_{h} U^{0}\right\|$ (in particular independent of $\left(M^{n}\right)$ ) such that for all $n, 1 \leq n \leq N_{T}$, for all $i, j, 0 \leq i, j \leq N_{h}$, and for all $\ell=1,2,3,4$,

$$
\left|\frac{\partial g}{\partial q_{\ell}}\left(x_{i, j},\left[D_{h} U^{n}\right]_{i, j}\right)\right| \leq C .
$$

From this, we see that for $\mu$ large enough but independent on $\left(M^{n}\right)$, the matrix corresponding to $I d+\Delta t\left(\mu I d+A^{n+1}\right)$ is a M-matrix, and is therefore invertible. The system of linear equations (74) has a unique solution.

Moreover, since $M^{0} \geq 0$ for all $n=0, \ldots, N_{T}$ and since $I d+\Delta t\left(\mu I d+A^{n+1}\right)$ is a M-matrix for all $n, 1 \leq n \leq N_{T}, \widetilde{M}^{n} \geq 0$ for all $n=0, \ldots, N_{T}$.

We are left with proving that $h^{2} \sum_{i, j} \widetilde{M}_{i, j}^{n}=1$, for all $n, 1 \leq n \leq N_{T}$. As in the proof of Theorem 1, we see that for two grid functions $W$ and $Z$, we have

$$
\begin{aligned}
\left(A^{n} W, Z\right)_{2}= & \nu \sum_{i, j}\left(D_{1}^{+} W\right)_{i, j}\left(D_{1}^{+} Z\right)_{i, j}+\nu \sum_{i, j}\left(D_{2}^{+} W\right)_{i, j}\left(D_{2}^{+} Z\right)_{i, j} \\
& +\sum_{i, j} W_{i, j}\left[D_{h} Z\right]_{i, j} \cdot \nabla_{q} g\left(x_{i, j},\left[D_{h} U^{n}\right]_{i, j}\right) .
\end{aligned}
$$

From (76) and (74), it can be proved by induction that if $h^{2}\left(M^{0}, 1\right)_{2}=1$, then the condition $h^{2}\left(\widetilde{M}^{n}, 1\right)_{2}=1$ holds for all $n, 1 \leq n \leq N_{T}$.

Step 6: existence of a fixed point of $\chi$ From the boundedness and continuity of the mapping $\Phi$, and from the fact that $g$ is $\mathcal{C}^{1}$, we obtain that $\chi$ is continuous. Therefore, we can apply Brouwer fixed point theorem and obtain that $\chi$ has a fixed point.

Conclusion Assuming that $M^{0}$ is such that $M^{0}>0$ and $h^{2}\left(M^{0}, 1\right)_{2}=1$, we have proved that the mapping $\chi$ has a fixed point that we call $\left(M^{n}\right)_{1 \leq n \leq N_{T}}$. Calling $\left(U^{n}\right)_{1 \leq n \leq N_{t}}=\Phi\left(\left(M^{n}\right)_{1 \leq n \leq N_{t}}\right)$, $\left(M^{n}\right)_{n=1 \ldots, N_{T}}$ and $\left(U^{n}\right)_{n=1 \ldots, N_{T}}$ satisfy $(65)$ and $(66)$.

Remark 6 Existence for problem (65)-(66) can also be obtained without (67) and when $V$ is a local operator, (see §3.4).

\subsection{A different fixed point strategy}

In the proof of Theorem 5, keeping the strategy unchanged up to Step 3, it is tempting to take $\mu=0$ in (73): in Step 4, we now have to find $\left(\widetilde{M}^{n}\right)_{1 \leq n \leq N_{T}}$ such that

$$
\frac{\widetilde{M}_{i, j}^{n+1}-\widetilde{M}_{i, j}^{n}}{\Delta t}-\nu\left(\Delta_{h} \widetilde{M}^{n+1}\right)_{i, j}-\mathcal{B}_{i, j}\left(U^{n+1}, \widetilde{M}^{n+1}\right)=0,
$$

with the initial condition $\widetilde{M}^{0}=M^{0}$, with $h^{2} \sum_{i, j} M_{i, j}^{0}=1$ and $M^{0} \geq 0$.

If (77) has a unique solution $\left(\widetilde{M}^{n}\right)_{1 \leq n \leq N_{T}} \in \mathcal{K}^{N_{T}}$, then we have a new mapping $\left(M^{n}\right)_{1 \leq n \leq N_{T}} \rightarrow$ 
$\left(\widetilde{M}^{n}\right)_{1 \leq n \leq N_{T}}$ which may have a fixed point. If it is the case, existence for (65) (66) will then be proved. We are going to show that this strategy is indeed feasible, under a mild assumption on the time step. In spite of this further assumption, this strategy is interesting because it uses a discrete weak maximum principle which will be useful for studying uniqueness.

Clearly (77) can be written

$$
\widetilde{M}^{n+1}+\Delta t A^{n+1} \widetilde{M}^{n+1}=\widetilde{M}^{n},
$$

with the notation defined above. We introduce the semi-norm $\||W|\|$ :

$$
\|\mid W\|^{2}=\sum_{i, j}\left(\left(D_{1}^{+} W\right)_{i, j}^{2}+\left(D_{2}^{+} W\right)_{i, j}^{2}\right) .
$$

From (75) and (51) we deduce that there exists a nonnegative constant $\sigma$ depending only of $\left\|D_{h} U^{0}\right\|_{\infty}$ (in particular independent of $\left(M^{n}\right)_{n=1, \ldots, N_{T}}$ and $n$ ) such that for all grid function $W$,

$$
\begin{aligned}
\left|\sum_{i, j} W_{i, j}\left[D_{h} W\right]_{i, j} \cdot \nabla_{q} g\left(x_{i, j},\left[D_{h} U^{n}\right]_{i, j}\right)\right| & \leq \frac{\nu}{2}\left\|\left|W\left\|\left.\right|^{2}+\sigma\right\| W \|_{2}^{2},\right.\right. \\
\left(A^{n} W, W\right)_{2} & \geq \frac{\nu}{2}\left\|\left|W\left\|\left.\right|^{2}-\sigma\right\| W \|_{2}^{2} .\right.\right.
\end{aligned}
$$

The latter estimate is a discrete Gårding inequality and implies that if $\sigma \Delta t<1$ then the system of linear equations (78) has a unique solution.

We now have to prove that $\widetilde{M}^{n} \geq 0$, for all $n, 1 \leq n \leq N_{T}$. For a grid function $W$, denote by $W^{+}$(resp. $W^{-}$) the grid function obtained by taking the positive (resp. negative) part of $W$ : $\left(W^{+}\right)_{i, j}=\left(W_{i, j}\right)^{+}$. Let us take $Z=-W^{-}$in (51): we have

$$
\begin{aligned}
-\left(A^{n} W, W^{-}\right)_{2}= & \nu \| W^{-}||^{2}+\frac{\nu}{h^{2}} \sum_{i, j}\left(W_{i, j} W_{i-1, j}\right)^{-}+\frac{\nu}{h^{2}} \sum_{i, j}\left(W_{i, j} W_{i, j-1}\right)^{-} \\
& +\sum_{i, j} W_{i, j}^{-}\left[D_{h} W^{-}\right]_{i, j} \cdot \nabla_{q} g\left(x_{i, j},\left[D_{h} U^{n}\right]_{i, j}\right) \\
& -\sum_{i, j} W_{i, j}^{+}\left[D_{h} W^{-}\right]_{i, j} \cdot \nabla_{q} g\left(x_{i, j},\left[D_{h} U^{n}\right]_{i, j}\right) .
\end{aligned}
$$

From (79), there exists a nonnegative constant $\sigma$ such that, for all grid function $W$,

$$
\left|\sum_{i, j} W_{i, j}^{-}\left[D_{h} W^{-}\right]_{i, j} \cdot \nabla_{q} g\left(x_{i, j},\left[D_{h} U^{n}\right]_{i, j}\right)\right| \leq \frac{\nu}{2}\left\|\left|W^{-}\right|\right\|^{2}+\sigma\left\|W^{-}\right\|_{2}^{2} .
$$

On the other hand, from the monotonicity of $g$, easy algebra shows that for all grid function $W$,

$$
-\sum_{i, j} W_{i, j}^{+}\left[D_{h} W^{-}\right]_{i, j} \cdot \nabla_{q} g\left(x_{i, j},\left[D_{h} U^{n}\right]_{i, j}\right) \geq 0
$$

From (80), (81) and (82), we see that for all grid functions $W$,

$$
-\left(A^{n} W, W^{-}\right)_{2} \geq\left.\frac{\nu}{2}\left\|W^{-}\right\|\right|^{2}-\sigma\left\|W^{-}\right\|_{2}^{2} .
$$


Similarly,

$$
\begin{aligned}
& -\left(\left(W^{n+1}-W^{n}\right),\left(W^{n+1}\right)^{-}\right)_{2}=\left(\left(\left(W^{n+1}\right)^{-}-\left(W^{n}\right)^{-}\right),\left(W^{n+1}\right)^{-}\right)_{2}+\sum_{i, j}\left(W_{i, j}^{n+1}\right)^{-}\left(W_{i, j}^{n}\right)^{+} \\
= & \frac{1}{2}\left\|\left(W^{n+1}\right)^{-}\right\|_{2}^{2}+\frac{1}{2}\left\|\left(W^{n+1}\right)^{-}-\left(W^{n}\right)^{-}\right\|_{2}^{2}-\frac{1}{2}\left\|\left(W^{n}\right)^{-}\right\|_{2}^{2}+\sum_{i, j}\left(W_{i, j}^{n+1}\right)^{-}\left(W_{i, j}^{n}\right)^{+} \\
\geq & \frac{1}{2}\left\|\left(W^{n+1}\right)^{-}\right\|_{2}^{2}+\frac{1}{2}\left\|\left(W^{n+1}\right)^{-}-\left(W^{n}\right)^{-}\right\|_{2}^{2}-\frac{1}{2}\left\|\left(W^{n}\right)^{-}\right\|_{2}^{2} .
\end{aligned}
$$

We deduce from (78), (83) and (84) that

$$
\left\|\left(\widetilde{M}^{n+1}\right)^{-}\right\|_{2}^{2}+\nu \Delta t\|\|\left(\widetilde{M}^{n+1}\right)^{-} \mid\left\|^{2}-2 \sigma \Delta t\right\|\left(\widetilde{M}^{n+1}\right)^{-}\left\|_{2}^{2} \leq\right\|\left(\widetilde{M}^{n}\right)^{-} \|_{2}^{2} .
$$

If $2 \sigma \Delta t<1$, then (85) implies that $\widetilde{M}^{n} \geq 0$ for all $n, 1 \leq n \leq N_{T}$ as soon as $M^{0} \geq 0$.

Remark 7 We have used a weak discrete maximum principle which does not require that the matrices corresponding to Id $+\Delta t A^{n}, n=1 \ldots, N_{T}$ be diagonal dominant. The latter may require a much more restrictive assumption on the time step, namely that $\alpha \frac{\Delta t}{h}<1$ for some positive $\alpha$. On the other hand, if the matrices corresponding to $I d+\Delta t A^{n}, n=1 \ldots, N_{T}$ are diagonal dominant, they are irreducible $M$-matrices, and we get the stronger property that $M^{0}>0$ implies $\widetilde{M}^{n}>0$ for all $n, 1 \leq n \leq N_{T}$.

The proof that $h^{2} \sum_{i, j} \widetilde{M}_{i, j}^{n}=1$, for all $n, 1 \leq n \leq N_{T}$ is obtained exactly as above. We thus have a new mapping $\chi: \mathcal{K}^{N_{T}} \mapsto \mathcal{K}^{N_{T}},\left(M^{n}\right)_{1 \leq n \leq N_{T}} \rightarrow\left(\widetilde{M}^{n}\right)_{1 \leq n \leq N_{T}}$. The existence of a fixed point for $\chi$ is proved as above.

\section{A finite difference scheme for problem (4)-(7)}

\subsection{Description of the scheme}

Given $M^{N_{T}}$, we choose the following scheme:

$$
\left\{\begin{aligned}
\frac{U_{i, j}^{n+1}-U_{i, j}^{n}}{\Delta t}-\nu\left(\Delta_{h} U^{n+1}\right)_{i, j}+g\left(x_{i, j},\left[D_{h} U^{n+1}\right]_{i, j}\right) & =\left(V_{h}\left[M^{n+1}\right]\right)_{i, j}, \\
\frac{M_{i, j}^{n+1}-M_{i, j}^{n}}{\Delta t}+\nu\left(\Delta_{h} M^{n}\right)_{i, j}+\mathcal{B}_{i, j}\left(U^{n}, M^{n}\right) & =0 \\
M_{i, j}^{n} & \geq 0
\end{aligned}\right.
$$

for $n=0, \ldots N_{T}-1$ and $0 \leq i, j<N_{h}$, with

$$
h^{2} \sum_{i, j} M_{i, j}^{n}=1, \quad \text { for } n=0, \ldots N_{T}-1,
$$

and

$$
U_{i, j}^{0}=\left(V_{0, h}\left(M^{0}\right)\right)_{i, j} \equiv V_{0}\left[m_{h}^{0}\right]\left(x_{i, j}\right),
$$

where $m_{h}^{0}$ is the piecewise constant function taking the value $M_{i, j}^{0}$ in the square $\left|x-x_{i, j}\right|_{\infty} \leq h / 2$. We have the analogue of Theorem 5 :

Theorem 6 We make the same assumptions as in Theorem 5, and we also assume that $V_{0}$ satisfies ( $\left.\mathbf{A}_{\mathbf{1}}\right)$ and $\left(\mathbf{A}_{\mathbf{2}}\right)$. If $M^{N_{T}} \geq 0$ and $\sum_{i, j} M_{i, j}^{N_{T}}=1$, then (86)-(88) has a solution. There exists a constant $C$ independent of $h$ and $\Delta t$ such that $\left\|D_{h} U^{n}\right\|_{\infty} \leq C$.

Proof. Similar to that of Theorem 5. 


\subsection{Uniqueness}

We slightly modify (86)-(88) in order to obtain uniqueness: we either replace the second equation in (86) for $n=0$ by $M^{0}=M^{1}$, or we replace (88) by

$$
U_{i, j}^{0}=\left(V_{0, h}\left(M^{1}\right)\right)_{i, j} .
$$

These modifications do not affect the consistency of the scheme. For brevity, we only discuss the second modification. Let $\left(U^{n}, M^{n}\right)_{n=0, \ldots, N_{T}}$ and $\left(\widetilde{U^{n}}, \widetilde{M}^{n}\right)_{n=0, \ldots, N_{T}}$ be two solutions of (86), (87), (89). We have

$$
\begin{aligned}
& \frac{\left(U^{n+1}-\widetilde{U}^{n+1}\right)_{i, j}-\left(U^{n}-\widetilde{U}^{n}\right)_{i, j}}{\Delta t}-\nu\left(\Delta_{h}\left(U^{n+1}-\widetilde{U}^{n+1}\right)\right)_{i, j} \\
& \quad+g\left(x_{i, j},\left[D_{h} U^{n+1}\right]_{i, j}\right)-g\left(x_{i, j},\left[D_{h} \widetilde{U}^{n+1}\right]_{i, j}\right)=\left(V_{h}\left[M^{n+1}\right]-V_{h}\left[\widetilde{M}^{n+1}\right]\right)_{i, j},
\end{aligned}
$$

for $n=0, \ldots N_{T}-1$. Multiplying by $Z_{i, j}^{n+1}$ and summing over all $i, j$ leads to

$$
\begin{aligned}
& \frac{\left(\left(U^{n+1}-\widetilde{U}^{n+1}\right)-\left(U^{n}-\widetilde{U}^{n}\right), Z\right)_{2}}{\Delta t}-\nu\left(\Delta_{h}\left(U^{n+1}-\widetilde{U}^{n+1}\right), Z\right)_{2} \\
& +\sum_{i, j}\left(g\left(x_{i, j},\left[D_{h} U^{n+1}\right]_{i, j}\right)-g\left(x_{i, j},\left[D_{h} \widetilde{U}^{n+1}\right]_{i, j}\right)\right) Z_{i, j} \\
= & \left(V_{h}\left[M^{n+1}\right]-V_{h}\left[\widetilde{M}^{n+1}\right], Z\right)_{2}, \quad n=0, \ldots N_{T}-1 .
\end{aligned}
$$

On the other hand, multiplying the second equation in (86) by $W_{i, j}$ and summing over all $i, j$ leads to

$$
\frac{\left(M^{n+1}-M^{n}, W\right)_{2}}{\Delta t}+\nu\left(M^{n}, \Delta_{h} W\right)_{2}-\sum_{i, j} M_{i, j}^{n}\left[D_{h} W\right]_{i, j} \cdot \nabla_{q} g\left(x_{i, j},\left[D_{h} U^{n}\right]_{i, j}\right)=0
$$

for $n=0, \ldots N_{T}-1$. From this and the similar equation satisfied by $\widetilde{M}^{n}$, we obtain

$$
\begin{aligned}
0 & =\frac{\left(\left(M^{n+1}-M^{n}\right)-\left(\widetilde{M}^{n+1}-\widetilde{M}^{n}\right), W\right)_{2}}{\Delta t}+\nu\left(\left(M^{n}-\widetilde{M}^{n}\right), \Delta_{h} W\right)_{2} \\
& -\sum_{i, j} M_{i, j}^{n}\left[D_{h} W\right]_{i, j} \cdot \nabla_{q} g\left(x_{i, j},\left[D_{h} U^{n}\right]_{i, j}\right)+\sum_{i, j} \widetilde{M}_{i, j}^{n}\left[D_{h} W\right]_{i, j} \cdot \nabla_{q} g\left(x_{i, j},\left[D_{h} \widetilde{U}^{n}\right]_{i, j}\right),
\end{aligned}
$$

for $n=0, \ldots N_{T}-1$. Taking $Z=M^{n+1}-\widetilde{M}^{n+1}$ in (91) for $n=0, \ldots N_{T}-1$ and $W=U^{n}-\widetilde{U}^{n}$ for $n=1, \ldots, N_{T}-1$ and summing leads to

$$
\begin{aligned}
0 & =\sum_{n=1}^{N_{T}-1}\left(V_{h}\left[M^{n}\right]-V_{h}\left[\widetilde{M}^{n}\right], M^{n}-\widetilde{M}^{n}\right)_{2}+\frac{1}{\Delta t}\left(V_{0, h}\left[M^{1}\right]-V_{0 h}\left[\widetilde{M}^{1}\right], M^{1}-\widetilde{M}^{1}\right)_{2} \\
& +\sum_{n=1}^{N_{T}-1} \sum_{i, j} M_{i, j}^{n}\left(g\left(x_{i, j},\left[D_{h} \widetilde{U}^{n}\right]_{i, j}\right)-g\left(x_{i, j},\left[D_{h} U^{n}\right]_{i, j}\right)-\left[D_{h}\left(\widetilde{U}^{n}-U^{n}\right)\right]_{i, j} \cdot \nabla_{q} g\left(x_{i, j},\left[D_{h} U^{n}\right]_{i, j}\right)\right) \\
& +\sum_{n=1}^{N_{T}-1} \sum_{i, j} \widetilde{M}_{i, j}^{n}\left(g\left(x_{i, j},\left[D_{h} U^{n}\right]_{i, j}\right)-g\left(x_{i, j},\left[D_{h} \widetilde{U}^{n}\right]_{i, j}\right)-\left[D_{h}\left(U^{n}-\widetilde{U}^{n}\right)\right]_{i, j} \cdot \nabla_{q} g\left(x_{i, j},\left[D_{h} \widetilde{U}^{n}\right]_{i, j}\right)\right),
\end{aligned}
$$

from $M^{N_{T}}=\widetilde{M}^{N_{T}}$ and (89). 
Theorem 7 We make the same assumptions as in Theorem 6. We assume furthermore that $g$ is convex w.r.t. to $\left(q_{1}, q_{2}, q_{3}, q_{4}\right)$, i.e. $\left(\mathbf{H}_{4}\right)$, and that the operators $V_{h}$ and $V_{0, h}$ are strictly monotone, i.e.

$$
\begin{aligned}
& \left(V_{h}[M]-V_{h}[\widetilde{M}], M-\widetilde{M}\right)_{2} \leq 0 \Rightarrow V_{h}[M]=V_{h}[\widetilde{M}] \\
& \left(V_{0, h}[M]-V_{0, h}[\widetilde{M}], M-\widetilde{M}\right)_{2} \leq 0 \Rightarrow V_{0, h}[M]=V_{0, h}[\widetilde{M}] .
\end{aligned}
$$

There exists a constant $\sigma \geq 0$ independent of $h$ such that if $2 \sigma \Delta t<1$, then (86), (87), (89) has a unique solution.

Proof. From the convexity of $g$ and the monotonicity assumptions on $V_{h}$ and $V_{0, h}$, the four terms in the right hand side of (94) vanish, and $V_{h}\left[M^{n}\right]=V_{h}\left[\widetilde{M}^{n}\right]$ for all $n=1, \ldots, N_{T}$, and $V_{0, h}\left[M^{1}\right]=V_{0, h}\left[\widetilde{M}^{1}\right]$. Since the scheme for $U^{n}$ is monotone, this implies that $U^{n}=\widetilde{U}^{n}$ for all $n=0, \ldots, N_{T}$. Let $\sigma$ be the constant of the Gårding inequality (79). Uniqueness for (78) (given $\left.U^{n}\right)$ yields that $M^{n}=\widetilde{M}^{n}$ for all $n=0, \ldots, N_{T}$.

\section{Numerical simulations}

\subsection{Long time approximation of the stationary problem}

As mentioned in the introduction, we consider a solution $(\widetilde{u}, \widetilde{m})$ of $(8)-(11)$ with the Cauchy data $\widetilde{m}_{0}$ and $\widetilde{u}_{0}$ defined on $\mathbb{T}^{2}, m_{0}$ being a probability measure. We e expect that there exist a $\mathcal{C}^{2}$ function $u$ on $\mathbb{T}^{2}$, a function $m$ in $W^{1, p}\left(\mathbb{T}^{2}\right)$ and a scalar $\lambda$ such that

$$
\lim _{t \rightarrow \infty} \widetilde{u}(t, x)-\lambda t=u(x), \quad \lim _{t \rightarrow \infty} \widetilde{m}(t, x)=m(x),
$$

and $\int_{\mathbb{T}^{2}} u=0$. If so, then $(u, m, \lambda)$ is a solution of (1)-(3).

Such long time approximations have been justified for the cell problem in the homogenization of Hamilton-Jacobi or Hamilton-Jacobi-Bellman equations, see for example [14], [1], [3]. This approach is close to the so-called eductive strategy in economy. In [6], O. Guéant studies the eductive stability on some examples where $V$ has not the monotony property used in Proposition 3 and justifies the approach.

The same long time approximation method may be used at the discrete level. The discrete version of (8)-(11) is (65)-(66) if an implicit Euler scheme is chosen. A semi-implicit linearized scheme can be used as well. Both schemes require the numerical solution of a linearized problem. Linearizing (65)-(66) needs careful computations and is not always possible. In such cases, an explicit method can be used.

Remark 8 In tests 1, 2, and 4, the Hamiltonian is of the form $H(x, p)=|p|^{2}+g(x)$. In such cases, as observed in [13], the system (1)-(3) is equivalent to a generalized Hartree equation. Indeed, introducing $\phi=\exp \left(-\frac{u}{\nu}\right)\left(\exp \left(-\frac{2 u}{\nu}\right)\right)^{-\frac{1}{2}}$ and taking $m=\phi^{2}$, the system reduces to $-\nu^{2} \Delta \phi-g \phi+\phi V\left[\phi^{2}\right]=\lambda \phi$ and $m$ can be written as a function of $u$.

\subsection{Results}

In all the problems considered below, the Hamiltonian is of the form $H(x, p)=\psi(x,|p|)$ and the discrete Hamiltonian is obtained via a Godunov scheme, i.e

$$
g\left(x, q_{1}, q_{2}, q_{3}, q_{4}\right)=\psi\left(x, \sqrt{\left(q_{1}^{-}\right)^{2}+\left(q_{3}^{-}\right)^{2}+\left(q_{2}^{+}\right)^{2}+\left(q_{4}^{+}\right)^{2}}\right) .
$$


A semi-implicit linearized scheme is used and the time step is progressively increased: when the asymptotic regime is reached, very long time steps $(\Delta t \sim 1000)$ have been used. On the other hand, if the condition $m^{n+1} \geq 0$ is violated, we start back from $\left(u^{n}, m^{n}\right)$ and decrease the time step $\delta t^{m}$.

Table 1 contains the data of the problems simulated below, i.e. the real number $\nu$, the Hamiltonian $H$ and the operator $V$.

Table 1: the real number $\nu$, the hamiltonian $H$ and the operator $V$

\begin{tabular}{|c|c|c|c|}
\hline Test & $\nu$ & $H(x, p)$ & $V[m](x)$ \\
\hline 1 & 1 & $\sin \left(2 \pi x_{2}\right)+\sin \left(2 \pi x_{1}\right)+\cos \left(4 \pi x_{1}\right)+|p|^{2}$ & $m^{2}(x)$ \\
\hline 2 & 0.01 & $\sin \left(2 \pi x_{2}\right)+\sin \left(2 \pi x_{1}\right)+\cos \left(4 \pi x_{1}\right)+|p|^{2}$ & $m^{2}(x)$ \\
\hline 3 & 0.1 & $\begin{array}{c}\sin \left(2 \pi x_{2}\right)+\sin \left(2 \pi x_{1}\right)+\cos \left(4 \pi x_{1}\right)+|p|^{\alpha} \\
\alpha=1.5,3,6,9\end{array}$ & $m^{2}(x)$ \\
\hline 4 & 0.1 & $\sin \left(2 \pi x_{2}\right)+\sin \left(2 \pi x_{1}\right)+\cos \left(4 \pi x_{1}\right)+|p|^{2}$ & $-\log (m(x))$ \\
\hline 5 & 0.1 & $\sin \left(2 \pi x_{2}\right)+\sin \left(2 \pi x_{1}\right)+\cos \left(4 \pi x_{1}\right)+|p|^{3 / 2}$ & $200\left((1-\Delta)^{-1}(1-\Delta)^{-1} m\right)(x)$ \\
\hline 6 & 0.001 & $\sin \left(2 \pi x_{2}\right)+\sin \left(2 \pi x_{1}\right)+\cos \left(4 \pi x_{1}\right)+|p|^{3 / 2}$ & $200\left((1-\Delta)^{-1}(1-\Delta)^{-1} m\right)(x)$ \\
\hline 7 & 0.001 & $\begin{array}{l}\sin \left(2 \pi x_{2}\right)+\sin \left(2 \pi x_{1}\right)+\cos \left(4 \pi x_{1}\right) \\
+(0.6+0.59 \cos (2 \pi x))|p|^{3 / 2}\end{array}$ & $200\left((1-\Delta)^{-1}(1-\Delta)^{-1} m\right)(x)$ \\
\hline 8 & 0.001 & $|p|^{2}$ & $4 \cos (4 \pi x)+m(x)$ \\
\hline
\end{tabular}

The contours of the potential $x \mapsto \sin \left(2 \pi x_{2}\right)+\sin \left(2 \pi x_{1}\right)+\cos \left(4 \pi x_{1}\right)$ are displayed on Figure 1.

\subsubsection{Test 1}

See Table 1 for the data of the problem. We first check that the long time approximation yields the expected asymptotic behavior. In Figure 2, we plot the graph of $\frac{h^{2}}{T} \sum_{i, j} U_{i, j}(t=T)$, when the mesh step is $h=1 / 50$; as $T$ tends to infinity, this quantity tends to a constant $\lambda_{h}$, as expected. Here, we find that $\lambda_{h} \simeq 0.9784$.

Here $V[m](x)=F(m(x))$ with $F(y)=y^{2}$ is a nondecreasing function. Such a function is used 


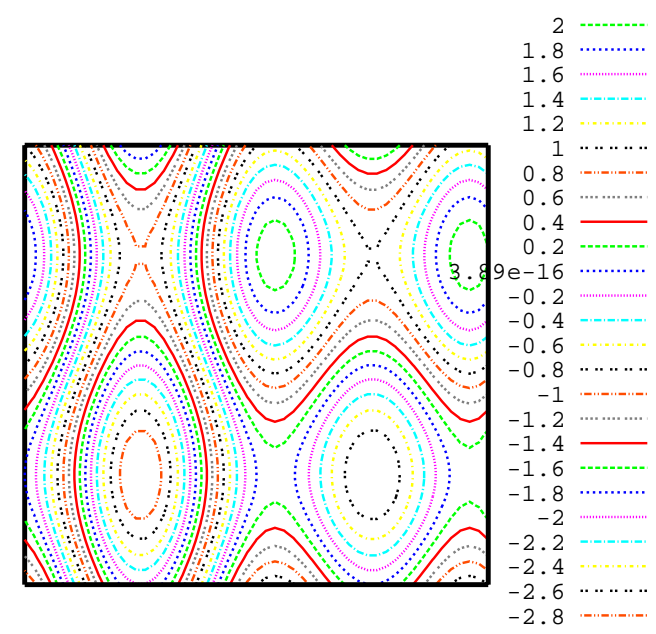

Figure 1: the contours of the potential $x \mapsto \sin \left(2 \pi x_{2}\right)+\sin \left(2 \pi x_{1}\right)+\cos \left(4 \pi x_{1}\right)$ used in tests 1-6.

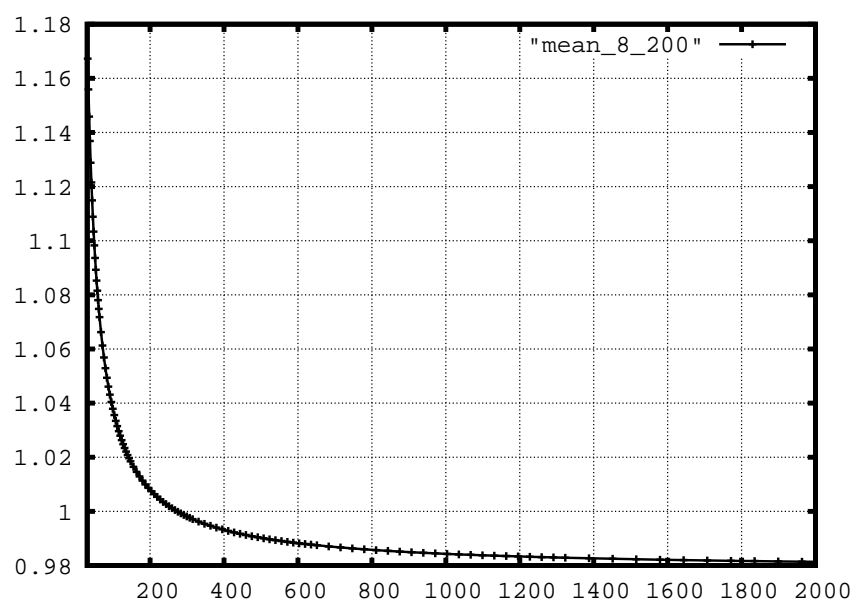

Figure 2: Test 1: graph of $\frac{h^{2}}{T} \sum_{i, j} U_{i, j}(t=T)$ for $T \leq 2000$, with $N_{h}=50$ 
to model repulsive cases when the players do not like to share their position with others. If $\nu$ is not too small, then the players positions should be well distributed, and the density $m$ should not be strongly localized. In Figure 3, we plot the contours of $u_{h}$ and $m_{h}$; we see indeed that $m_{h}$ is supported in the whole domain $\mathbb{T}^{2}$. Moreover, from Remark 8 and since $\nu$ is large enough, $m$ is a smooth decreasing function of $u$. This explains why the contour plots of $u_{h}$ and $m_{h}$ have the same aspect.
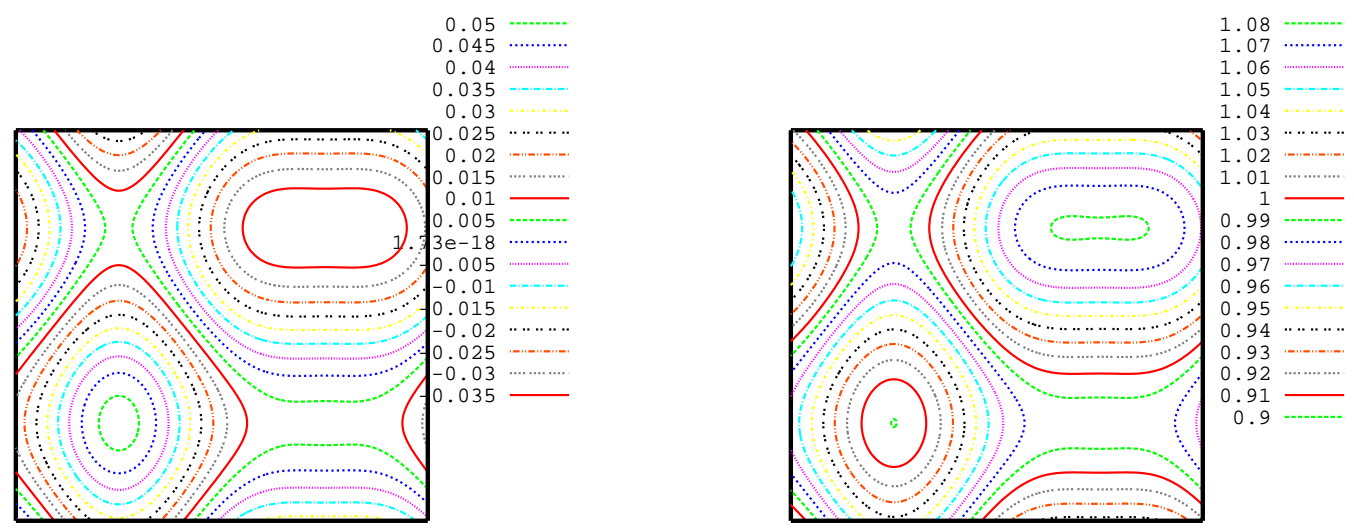

Figure 3: Test 1: the contours of $u_{h}$ (left) and $m_{h}$ (right), with $N_{h}=200$

Finally, in order to estimate the rate of convergence as $h$ tends to 0 , we compare the solutions with the one obtained with $N_{h}=200$ (considered as a fine grid). We compute the errors in the maximum norm. The graphs of the errors are displayed in Figure 4. The convergence looks linear.

\subsubsection{Test 2}

See Table 1 for the data of the problem. Here the value of $\nu=0.01$ is small, so the case is close to the deterministic limit.

As in Test 1, the solution of the discrete non stationary problem has the expected behavior for large times. In Figure 5, we plot the graph of $\frac{h^{2}}{T} \sum_{i, j} U_{i, j}(t=T)$, when the mesh step is $h=1 / 50$; as $T$ tends to infinity, this quantity tends to a constant $\lambda_{h}$, as expected. Here, we find that $\lambda_{h} \simeq 1.1885$.

In Figure 6, we plot the contours of $u_{h}$ and $m_{h}$. Note that the supports of $\nabla u_{h}$ and of $m_{h}$ tend to be disjoint for such a small values of $\nu$. This is coherent with the results concerning the deterministic limit in [13], see also $\S 6.2 .8$ below for more details. This test confirms the fact that the method is robust for small values of $\nu$.

\subsubsection{Test 3}

In Figure 7, we compare $m_{h}$ for different values of $\alpha$ (see Table 1). We see that the variations of $m_{h}$ become stiffer as $\alpha$ grows. 

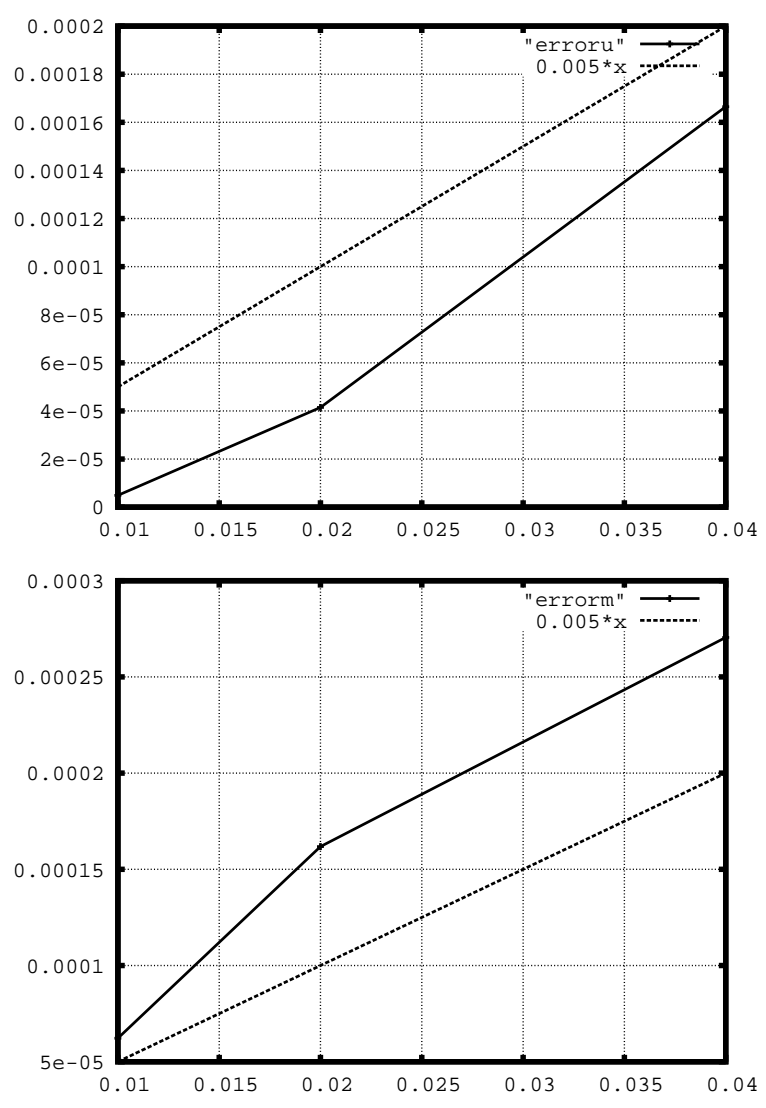

Figure 4: Test 1: error with respect to the discrete solutions obtained on a $200 \times 200$ grid as a function of $h$. Top: error for $u$, bottom: error for $m$

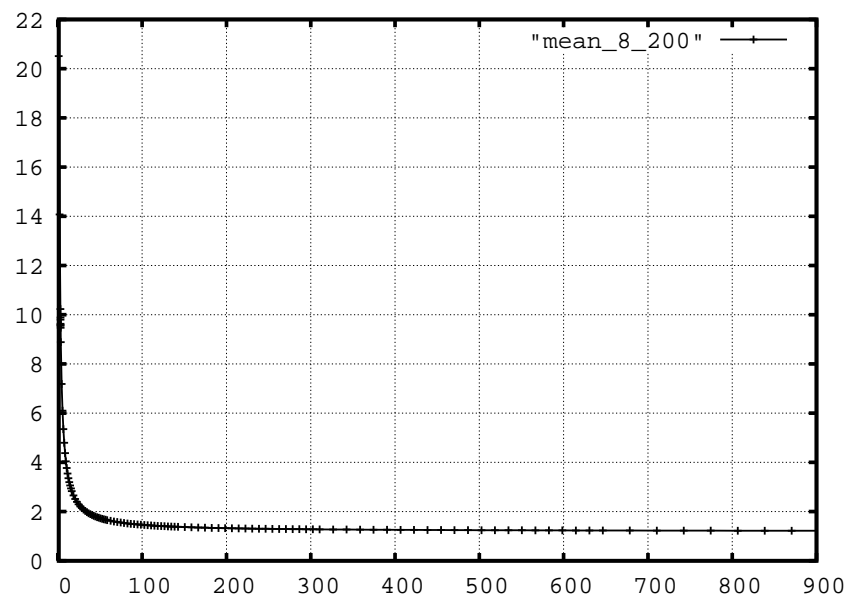

Figure 5: Test 2: graph of $\frac{h^{2}}{T} \sum_{i, j} U_{i, j}(t=T)$ for $T \leq 900$, with $N_{h}=50$ 

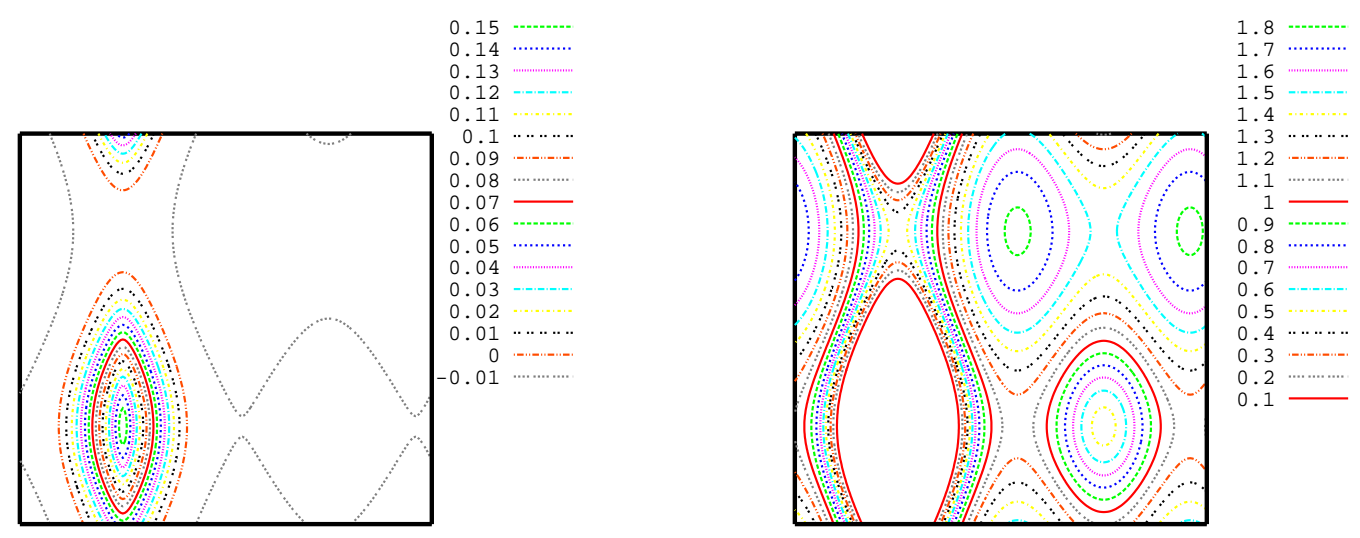

Figure 6: Test 2: the contours of $u_{h}$ (left) and $m_{h}$ (right), with $N_{h}=200$
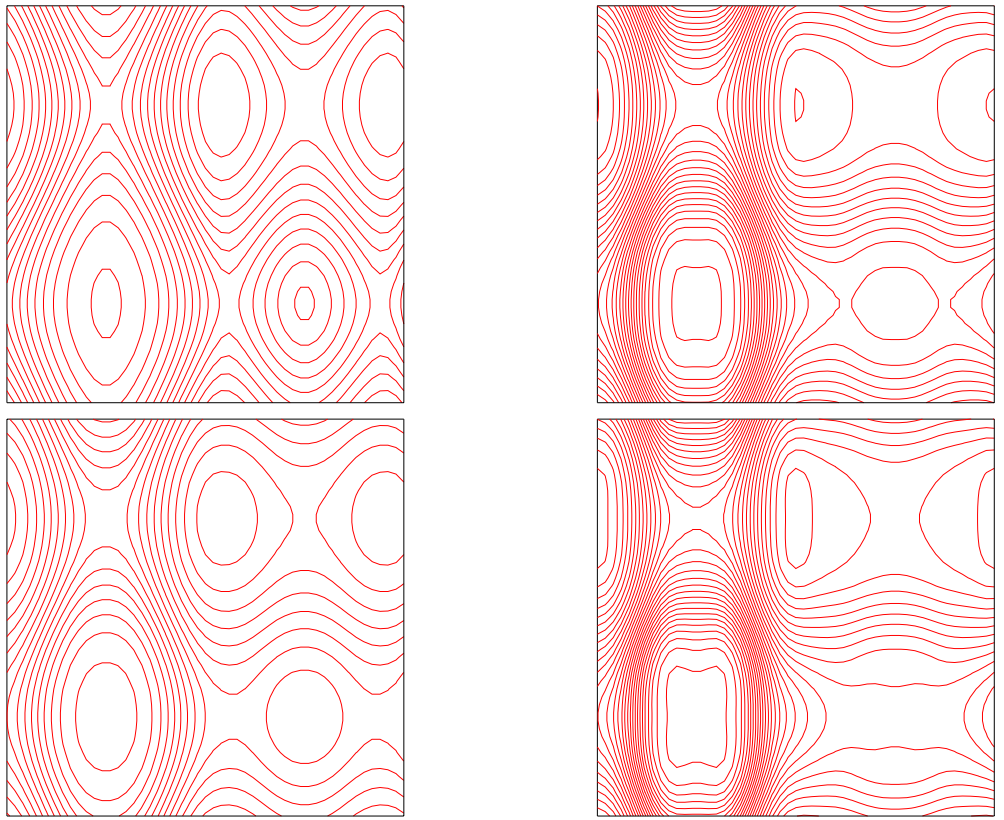

Figure 7: Test 3: contours of $m_{h}$ : comparison for $\alpha=1.5$ (top-left), $\alpha=3$ (bottom-left), $\alpha=6$ (top-right), $\alpha=9$ (bottom-right) 


\subsubsection{Test 4}

See Table 1 for the data of the problem. Here $V[m](x)=F(m(x))$ with $F(y)=-\log (y)$. By contrast with tests 1 and $2, F$ is a decreasing function. Such a function $F$ is used to describe situations when the agents are gregarious, i.e. they like to all be in the same position. O. Guéant proved results concerning the eductive stability in close cases, see [6]. Indeed, as shown in Figure 8, the solution of the discrete non stationary problem has the expected behavior for large times.

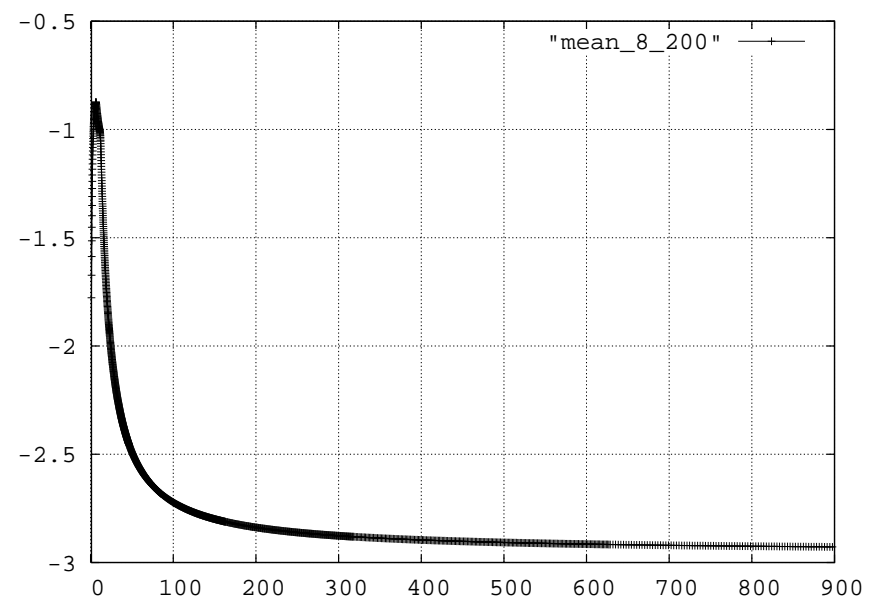

Figure 8: Test 4: graph of $\frac{h^{2}}{T} \sum_{i, j} U_{i, j}(t=T)$ for $T \leq 900$, with $N_{h}=100$

In Figure 9, we plot the contours of $u_{h}$ and $m_{h}$. Note that the measure $m_{h}$ concentrates near the minimum of $u_{h}$, i.e. the players take positions close to each other.
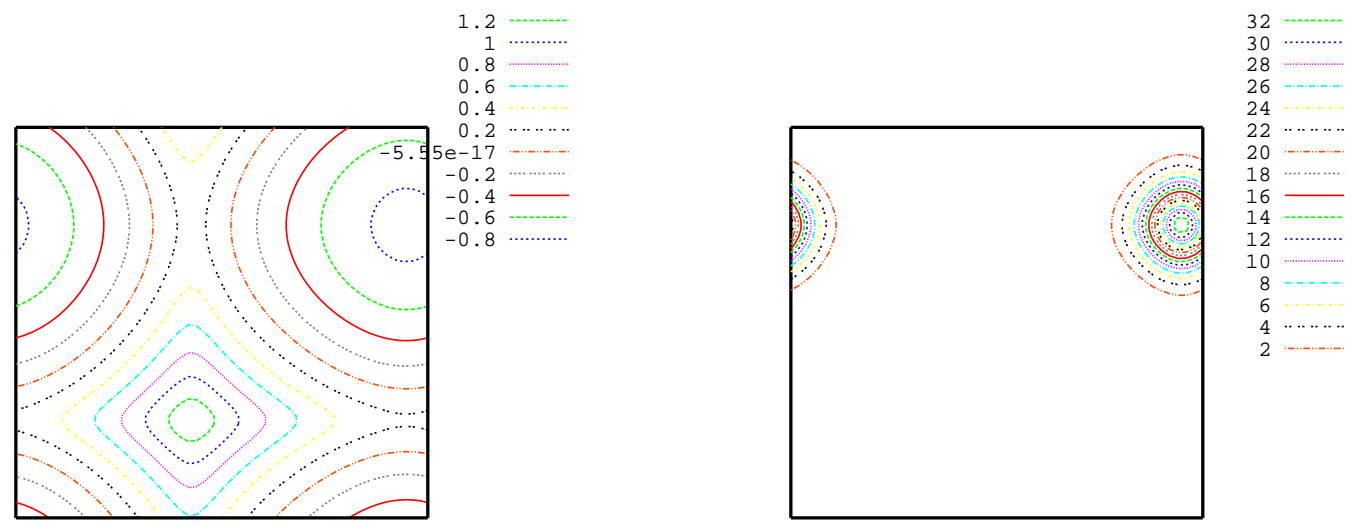

Figure 9: Test 4: the contours of $u_{h}$ (left) and $m_{h}$ (right), with $N_{h}=100$ 


\subsubsection{Test 5}

See Table 1 for the data of the problem. By contrast with the previous cases, the operator $V$ is nonlocal. At the discrete level, applying $V$ is done by solving a system of linear equations. Alternatively, a method based on fast Fourier transform could be used. In Figure 10, we plot the contours of $u_{h}$ and $m_{h}$.
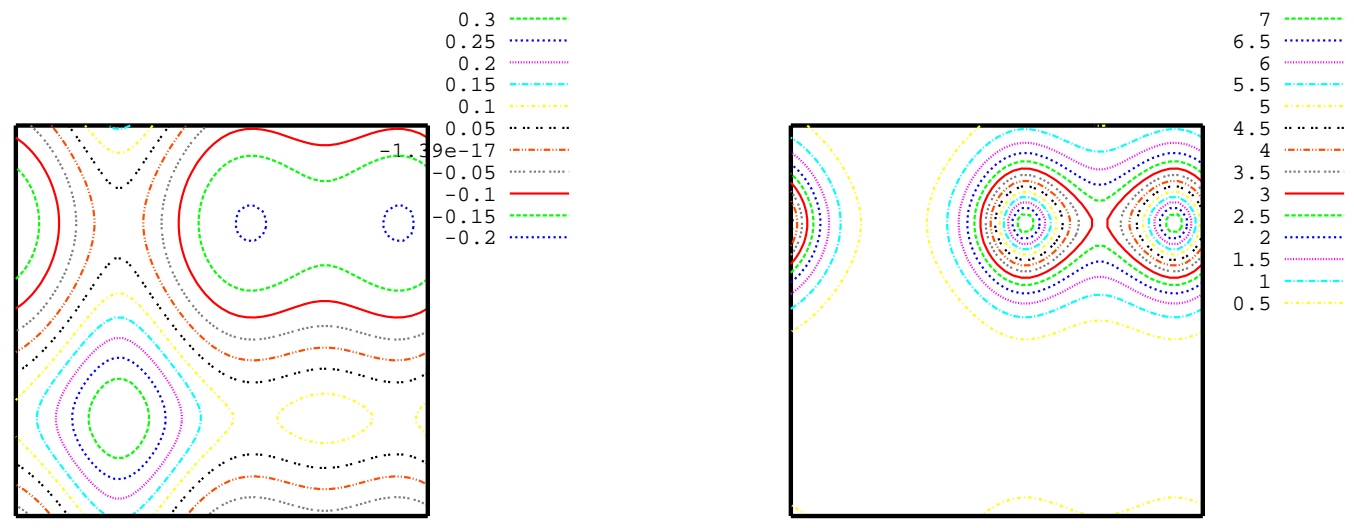

Figure 10: Test 5: the contours of $u_{h}$ (left) and $m_{h}$ (right), with $N_{h}=100$

\subsubsection{Test 6}

See Table 1 for the data of the problem. Compared to Test 5, we keep everything unchanged, except that we now take $\nu=0.001$. In Figure 11, we plot the graphs of $u_{h}$ and $m_{h}$. We see that $u_{h}$ is not better than Lipschitz continuous and that $m_{h}$ is close to a sum of two Dirac masses located at the minima of $u_{h}$.

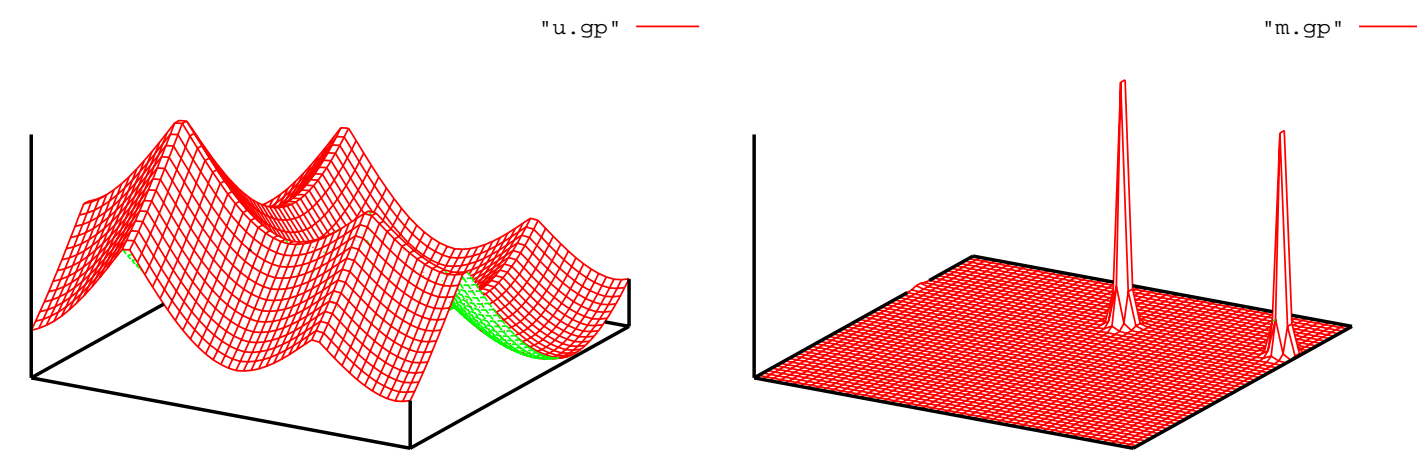

Figure 11: Test 6: the graphs of $u_{h}$ (left) and $m_{h}$ (right) 


\subsubsection{Test 7}

See Table 1 for the data of the problem. Compared to Test 5, we keep everything unchanged, except that the Hamiltonian strongly couples $x$ and $p$. In Figure 12, we plot the contours of $u_{h}$ and $m_{h}$.
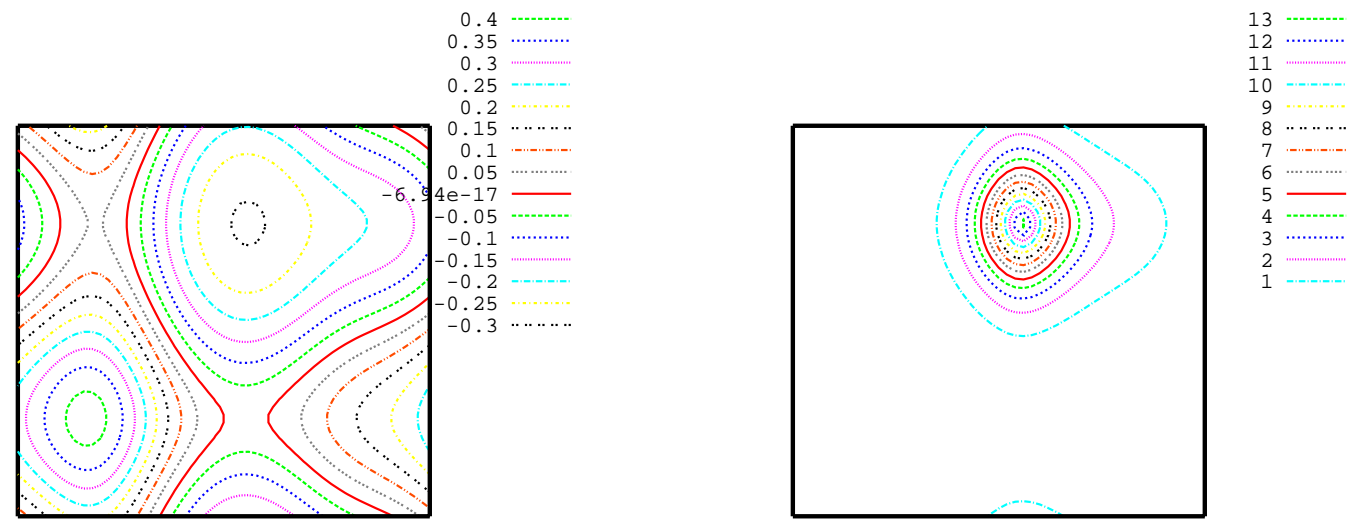

Figure 12: Test 7: the contours of $u_{h}$ (left) and $m_{h}$ (right), with $N_{h}=100$

\subsubsection{Test 8: the deterministic limit}

See Table 1 for the data of the problem. In [13], it is proved that if $H(x, p) \geq H(x, 0)=0$ and if $V[m]=F(m)+f_{0}(x)$ where $F^{\prime}>0$, then calling $\lambda_{\nu} u_{\nu}, m_{n} u$ the solution of (1), (2), (3),

$$
\lim _{\nu \rightarrow 0}\left(\lambda_{\nu}, m_{\nu}\right)=(\widetilde{\lambda}, \widetilde{m})
$$

where

$$
\widetilde{m}(x)=\left(F^{-1}\left(\widetilde{\lambda}-f_{0}(x)\right)\right)^{+} \quad \text { and } \quad \int_{\mathbb{T}^{2}} \widetilde{m} d x=1 .
$$

To illustrate this property, we choose

$$
\nu=0.001, \quad H(x, p)=|p|^{2}, \quad V[m](x)=4 \cos (4 \pi x)+m .
$$

In Figure 13, we plot the graphs of $u_{h}$ and $m_{h}$. We obtain that $\widetilde{m}(x) \approx(\widetilde{\lambda}-4 \cos (4 \pi x))^{+}$, as expected. We also see that the supports of $\nabla \widetilde{u}$ and of $\widetilde{m}$ are disjoint.

Acknowledgement It is a pleasure to thank F. Camilli for a very helpful discussion and for letting us read his work in preparation.

\section{References}

[1] Y. Achdou, F. Camilli, and I. Capuzzo Dolcetta. Homogenization of Hamilton-Jacobi equations: numerical methods. Math. Models Methods Appl. Sci., 18(7):1115-1143, 2008. 

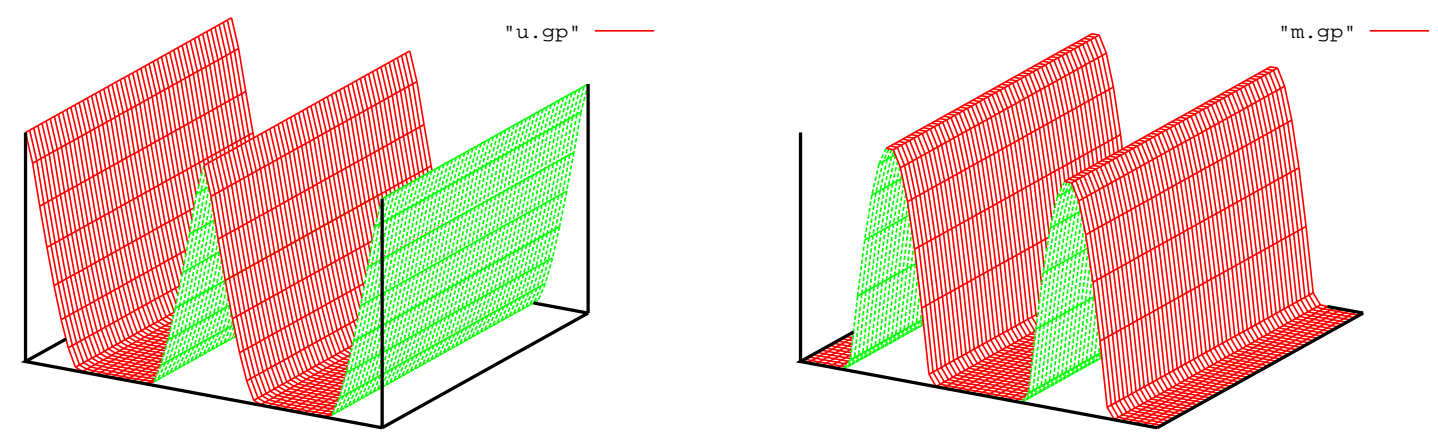

Figure 13: Test 8: the contours of $u_{h}$ (left) and $m_{h}$ (right), with $N_{h}=100$

[2] Y. Achdou and I. Capuzzo Dolcetta. Mean field games: Numerical methods for the finite horizon problem. in preparation.

[3] F. Camilli and C. Marchi. Rates of convergence in periodic homogenization of fully nonlinear uniformly elliptic pdes. in preparation.

[4] B. Cockburn and J. Qian. Continuous dependence results for Hamilton-Jacobi equations. In Collected lectures on the preservation of stability under discretization (Fort Collins, CO, 2001), pages 67-90. SIAM, Philadelphia, PA, 2002.

[5] D. Gomes, J. Mohr, and R.R. Souza. Discrete mean field games. preprint.

[6] O. Guéant. A reference case for mean field games models. Technical report, CEREMADE, U. Paris Dauphine, 2008.

[7] N. V. Krylov. A priori estimates of smoothness of solutions to difference Bellman equations with linear and quasi-linear operators. Math. Comp., 76(258):669-698 (electronic), 2007.

[8] H. J. Kuo and N. S. Trudinger. Linear elliptic difference inequalities with random coefficients. Math. Comp., 55(191):37-53, 1990.

[9] H. J. Kuo and N. S. Trudinger. Discrete methods for fully nonlinear elliptic equations. SIAM J. Numer. Anal., 29(1):123-135, 1992.

[10] A. Lachapelle, J. Salomon, and G. Turinici. A monotonic algorithm for a mean field games model in economics. Technical report, CEREMADE, U. Paris Dauphine, 2009.

[11] J.-M. Lasry and P.-L. Lions. Jeux à champ moyen. I. Le cas stationnaire. C. R. Math. Acad. Sci. Paris, 343(9):619-625, 2006.

[12] J.-M. Lasry and P.-L. Lions. Jeux à champ moyen. II. Horizon fini et contrôle optimal. C . R. Math. Acad. Sci. Paris, 343(10):679-684, 2006.

[13] J.-M. Lasry and P.-L. Lions. Mean field games. Jpn. J. Math., 2(1):229-260, 2007.

[14] P.L. Lions, G. Papanicolaou, and S.R.S. Varadhan. Homogenization of Hamilton-Jacobi equations. unpublished. 